\title{
Sex disparate gut microbiome and metabolome perturbations precede disease progression in a mouse model of Rett syndrome
}

\author{
Kari Neier \\ University of California, Davis \\ Tianna Grant \\ University of California, Davis \\ Rebecca Palmer \\ University of California, Davis \\ Demario Chappell \\ University of California, Davis \\ Sophia Hakam \\ University of California, Davis \\ Kendra Yasui \\ University of California, Davis \\ Matt Rolston \\ University of California, Davis \\ Matthew Settles \\ UC Davis Genome Center \\ Samuel Hunter \\ University of California Davis Genome Center \\ Blythe Durbin-Johnson \\ UC Davis \\ Janine LaSalle ( $\sim$ jmlasalle@ucdavis.edu ) \\ University of California, Davis https://orcid.org/0000-0002-3480-2031 \\ Dag Yasui \\ UC Davis School of Medicine https://orcid.org/0000-0003-1178-988X
}

Article

Keywords: Gut Dysbiosis, Altered Metabolism, MECP2, Neuromotor Phenotypes, Lipid Deficiencies, Clostridia

Posted Date: May 28th, 2021

DOl: https://doi.org/10.21203/rs.3.rs-478134/v1

License: () (i) This work is licensed under a Creative Commons Attribution 4.0 International License. Read Full License

Version of Record: A version of this preprint was published at Communications Biology on December 1st, 2021. See the published version at https://doi.org/10.1038/s42003-021-02915-3. 


\section{Abstract}

Rett syndrome (RTT) is a severe regressive neurodevelopmental disorder in girls, characterized by multisystem complications including gut dysbiosis and altered metabolism. While RTT is known to be caused by mutations in the X-linked gene $M E C P 2$, the intermediate molecular pathways of progressive disease phenotypes are unknown. Mecp 2 knockout rodents used to model RTT pathophysiology in most prior studies have been male. Thus, we utilized a patient-relevant mouse model of RTT to longitudinally profile the gut microbiome and metabolome across disease progression in both sexes. Fecal metabolite alterations in Mecp2e1 mutant females occurred prior to onset of neuromotor phenotypes and correlated with lipid deficiencies in brain, results not observed in males. Females also displayed altered gut microbial communities, including those belonging to Clostridia, that were more consistent with RTT patients than males. These findings suggest new potential therapeutic targets for RTT and demonstrate the importance of further study in female animal models.

\section{Introduction}

Rett syndrome (RTT, OMIM \#312750) is one of the most common genetic causes of intellectual disabilities in females, affecting 1 in 10,000 births ${ }^{1}$. RTT is an X-linked dominant disorder that is predominantly caused by mutations in $M E C P 2^{2}$, a gene encoding Methyl-CpG Binding Protein 2 (MeCP2). Although RTT is a monogenic disorder, its molecular pathogenesis remains poorly understood, particularly in Mecp2 heterozygous females. Further complexities of RTT are likely due to pleiotropic molecular functions and ubiquitous expression of MeCP2. MeCP2 was originally characterized as a methyl $\mathrm{CpG}$ binding protein and transcriptional repressor ${ }^{3}$. More recently, MeCP2 has been shown to; 1 ) bind to additional nucleotide motifs across the genome $4-8,2$ ) activate as well as repress gene transcription 9,10 including microRNAs (miRNA) ${ }^{11,12}, 3$ ) regulate alternative splicing ${ }^{13}$, and 4) nucleate higher-order chromatin organization ${ }^{14}$. Furthermore, X-chromosome inactivation results in mosaic expression of MECP2 mutations in females, and cells expressing mutant MECP2 have non-cell autonomous, or "bad neighborhood" effects, negatively impacting nearby wild-type cells in Rett females ${ }^{15-17}$. RTT patients are predominantly heterozygous females because spontaneous de novo mutations occur more frequently on the paternal X-chromosome ${ }^{18}$ and early lethality occurs in boys with RTT-causing MECP2 mutations. Despite the vast majority of RTT patients being female, the majority of gene therapy and other preclinical studies in animal models of RTT have been conducted in male mice hemizygous for Mecp2 deletion. The complexities of RTT and MeCP2 function have resulted in significant challenges for developing safe and effective gene therapies ${ }^{19}$, and it is unclear whether gene therapies given to existing RTT patients would effectively mitigate systemic manifestations of the disease. Thus, well characterized, gene-relevant female mosaic mouse models are needed to uncover underlying molecular, cellular, and physiological intermediate phenotypes in the pathophysiology of RTT in order to provide insights into potential therapies.

Individuals with RTT are born with an apparently normal phenotype, with distinct symptoms emerging at 6-18 months of age that include regression of motor and language skills, seizures, microcephaly, loss of purposeful hand movements, breathing abnormalities and severe cognitive impairments ${ }^{20,21}$. The neurologic features of RTT in conjunction with the importance of MeCP2/MECP2 in neuronal development and function in adulthood have prompted intense research focus on the nervous system in RTT patients and mouse models. However, RTT has been increasingly recognized as a multi-system disorder likely due to the expression of $M E C P 2$ in almost all cell types. For example, approximately two-thirds to four-fifths of RTT patients frequently report gastrointestinal disturbances ${ }^{22,23}$, and half of RTT patients have high serum cholesterol, triglycerides, and/or LDL cholesterol ${ }^{24}$. Male, Mecp2-/y hemizygous mice with deletion of Mecp2 exons 3 and 4 (Mecp2-null) showed severe defects in the colonic epithelium ${ }^{25}$, and an $\mathrm{N}$-ethyl-N-nitrosourea mutant suppressor screen revealed a significant role for Mecp2 in lipid metabolism ${ }^{26}$.

The gut microbiome maintains physiological function of the GI tract, energy metabolism, the immune system, as well as modulating brain and behavior ${ }^{27}$. Furthermore, changes in bacterial genus abundance correlate with neurologic phenotypes in schizophrenia ${ }^{28}$, major depressive disorder ${ }^{29}$ and Parkinson's disease ${ }^{30}$. Extensive cross-talk between the gut and the nervous system involving gut microbes and the metabolites they produce ${ }^{31-33}$ suggests a potential role for the gut microbiome in RTT. Recent clinical studies have found that the gut microbiome is perturbed in RTT patients ${ }^{34,35}$. However, whether the altered microbiota in RTT patients is simply a consequence of their altered diet and feeding behavior or an important contributor to 
regression is currently unknown. Thus, examination of the gut microbiome and metabolome in a female mouse model of RTT throughout the course of disease progression would provide critical insights into RTT pathophysiology.

RTT is a progressive neurologic disorder with age-specific manifestations. RTT is divided into four stages: early stagnation, rapid regression, stabilization, and late motor deterioration ${ }^{36}$. In addition, motor skill defects, mood, and gastrointestinal problems vary with increased age in RTT patients ${ }^{23,37}$. Mouse models of RTT recapitulate progression of motor, behavioral, and metabolic phenotypes. Both male Mecp $2^{-/ y}$-null and female $M e c p 2^{-1+}$ heterozygous mutant mice exhibit age- and sex-specific increases in body weight, gait alterations, reduced anxiety behaviors, and decreased performance on beam walking and rotarod tasks ${ }^{38-40}$. Age-related signatures of RTT can also be detected at the neuronal level. For example, GABA signaling is decreased in an agespecific manner in $M e c p 2^{-/ y}$ null males ${ }^{41}$, and dendritic spine density in $M e c p 2^{-/ y}$ relative to Mecp2 ${ }^{+/ y}$ control males is progressively decreased with age reflecting age-related behavioral phenotypes ${ }^{42}$. Furthermore, neuronal MeCP2 expression levels increase with age ${ }^{17,43}$. While there is substantial evidence of biochemical and biomolecular age-related signatures of RTT in the brain, molecular signatures of gastrointestinal and metabolic disease progression have not yet been thoroughly examined.

We sought to characterize gastrointestinal and metabolic molecular signatures of RTT disease progression at the gut-brain interface by simultaneously evaluating the fecal microbiome and metabolome longitudinally across symptom progression in an established construct-relevant mouse model of RTT, a Mecp2-e1 isoform-specific knock-in mutation found in human RTT patients

38,40. We examined both male hemizygous Mecp2-e $T^{-/ y}$ mice and female mosaic heterozygous $M e c p 2-e T^{-/+}$mice to identify sexspecific differences in RTT disease progression. Fecal collection allowed us to longitudinally analyze microbiome and metabolome profiles from the same mice across disease progression, and we utilized weighted network construction analyses to integrate molecular and phenotypic data. Lastly, we measured the brain lipidome in male and female mutant Mecp2-e1 mice to determine whether changes in the gut microbiome and metabolome were associated with altered lipid profiles in the brain. These findings demonstrate that pathophysiology and progression of RTT is substantially different in females than in males and suggest that changes in the microbiome and metabolism of the gastrointestinal tract influence progression of overt neurological, motor, and metabolic symptoms in RTT, providing new avenues for potential treatments and therapies.

\section{Results}

\section{Mice deficient in MeCP2e1 display sexually dimorphic progression of neurological, motor, and body weight phenotypes}

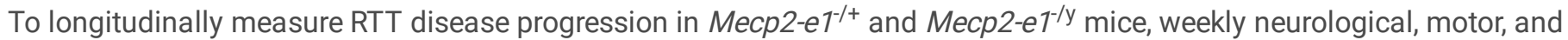
metabolic phenotyping assessments were carried out on mutant and wild-type littermates between 5- 6 and 19 weeks of age for females and 5-6 and 16 weeks of age for males. Male Mecp2-e $1^{-1 / y}$ mice began to experience increased morbidity and mortality at approximately 16 weeks of age, so measurements ceased at 16 weeks and mice were euthanized as required by IACUC protocol. Neurological phenotyping scores were assigned based on a system previously used to assess disease progression in RTT mouse models ${ }^{40,44}$, motor phenotyping of gait was performed by footprint analysis, and gross metabolic phenotype was assessed by body weight measurements.

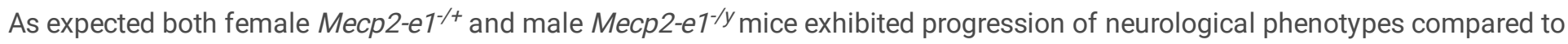
wild-type littermates (longitudinal $p<0.0001$, both sexes), however onset was earlier and disease severity was greater for males than for females (Figure 1a), as previously described ${ }^{38}$. The earliest age at which there was a statistically significant increase in neuro-phenotyping scores was at 10 weeks for females and at 7 weeks for males, with males displaying greater severity of neurological symptoms, as expected.

In congruence with neurological motor phenotyping outcomes, gait analyses performed from 6 and 17 weeks in females and 6 and 15 weeks in males indicate that while both female and male Mecp2-e1 mutant mice exhibited progressive motor phenotypes compared to Mecp2 wild-type control littermates, symptom onset was earlier and severity was greater for males (Figure 1b). In females, Mecp2-e $1^{-/+}$mutants had larger foot overlap distances between the front and hind legs than $M e c p 2^{+/+}$littermates at 6 and 9 weeks, with persistent differences beginning at 11 weeks of age, in contrast to the persistence of this phenotype from 6 to 15 weeks in mutant males (Figure 1b). The differences between overlap distances in Mecp2 mutant compared to Mecp2 wild-type 
littermates were also greater in males, specifically $0.93+/-0.18 \mathrm{~cm}$ at 13 weeks of age compared to $0.54+/-0.16 \mathrm{~cm}$ at 16 and 17 weeks of age in females. In addition, stride length and distance between hind legs were also impaired in Mecp2-e1 mutant mice, demonstrating earlier onset and severity in males compared to females (Supplemental Figure 1).

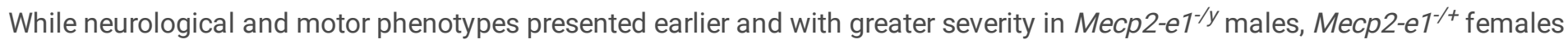
exhibited earlier onset of progressive body weight gain throughout disease course (Figure 1c). Longitudinally, female Mecp2-e $1^{T^{/+}}$ mice weighed more than Mecp2-e $1^{+/+}$littermates, and a non-significant trend was observed in the same increased direction for

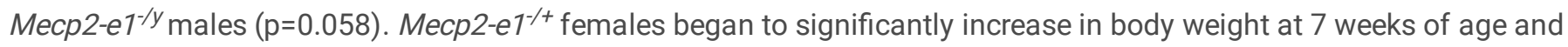
continued to gain weight through 19 weeks of age, with female Mecp2-e1/t+ mice weighing an average of $13.5+/-2.8$ grams more

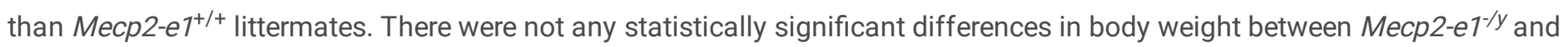
Mecp2-e $1^{+/ y}$ males at any age. Together, these longitudinal phenotyping analyses complement previous studies in the disease relevant Mecp2-e1 RTT mouse model by demonstrating progressive yet distinct onset differences in neurological, motor, and metabolic phenotypes between sexes ${ }^{38,40}$.

\section{Mice deficient in MeCP2e1 display sex-specific alterations in the fecal microbiome beginning early in postnatal life}

To identify microbial signatures of RTT disease progression in the gastrointestinal tract, we carried out and analyzed 16S sequencing on fecal samples collected longitudinally on a weekly basis between 5 and 19 weeks of age for female Mecp2-e $1^{-1+}$ and Mecp2-e $1^{+/+}$littermates and between 5 and 16 weeks of age for male Mecp2-e T $^{-/ y}$ and Mecp2-e $1^{+/ y}$ mice. Illumina MiSeq sequencing reads were resolved to amplicon sequence variants (ASVs) and classified by Phylum, Class, Order, Family, Genus, and Species. Rarefaction curves indicated that at 250 ASVs detected, the majority of diversity in the population had been sampled (Supplemental Figure 2); only 6 of 787 total samples did not have at least 250 ASVs and were removed from downstream analysis.

In general, ASVs identified from all fecal samples were primarily from the Firmicutes and Bacteroidetes phyla, which together made up over $95 \%$ of ASVs, with the remainder lower relative abundance phyla made up of Actinobacteria, Cyanobacteria, Fusobacteria, Patescibacteria, Proteobacteria, Tenericutes, and Verrucomicrobia (Supplemental Figure 3), consistent with the typical adult mouse gut microbiome ${ }^{45}$. However, mutant Mecp2-e1 mice had sex-specific, differential relative abundances of Firmicutes, Bacteroidetes, Actinobacteria, Tenericutes, and Verrucomicrobia longitudinally across disease course (Table 1). Longitudinally, female Mecp2-e $1^{-/+}$mutants had increased relative abundance of Firmicutes by $3.7 \%$ and decreased relative abundance of Bacteroidetes by 3.4\% compared to Mecp2-e $1^{+/+}$littermates. In contrast, males had the opposite genotype-related shifts in relative Firmicutes and Bacteroidetes abundance, specifically a decreased relative abundance of Firmicutes by $5.1 \%$ and increased relative abundance of Bacteroidetes by $5.2 \%$ in Mecp2-e $1^{-/ y}$ versus Mecp2-e $1^{+/ y}$ littermates. Notably, the expansion of Firmicutes observed in female Mecp2-e1//+ mutants was due to increased relative abundance of Clostridia (Supplemental Table 1), a class of microbes that regulate the gut-brain axis ${ }^{46}$. In addition, female Mecp2-e $1^{-/+}$mutants had $0.28 \%$ lower relative

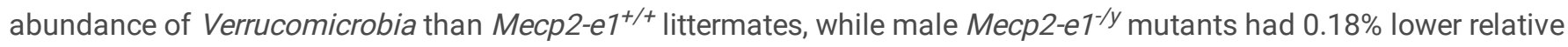
abundance of Actinobacteria and $0.30 \%$ higher relative abundance of Tenericutes compared to Mecp2-e1 $1^{+/ y}$ littermates. In addition to sex-specific relationships between genotype and relative abundance of various microbial Phyla longitudinally, there were also several significant relationships cross-sectionally (Supplemental Figure 4).

Microbiome diversity is often considered an indicator of gut health, and studies have suggested that the fecal microbiome is less diverse in RTT patients ${ }^{34,35}$. Surprisingly, we found that in females, two measures of microbiota diversity, the Shannon and Chao indices, were increased longitudinally across disease progression in mutant Mecp2-e1 mice relative to wild-type littermates (Table 1). There were no significant differences in diversity scores between mutant and wild-type males. Cross-sectionally, the most pronounced differences in microbiota diversity scores were at 12 and 16 weeks in Mecp2-e $1^{-1+}$ females (Supplemental Figure 5). Despite the lack of statistically significant differences in longitudinal diversity scores in Mecp2-e $1^{-1 / y}$ males, cross-sectional analyses identified significantly different diversity at 5, 6, and 8 weeks of age between Mecp2-e $1^{-1 / y}$ and Mecp2-e $1^{+/ y}$ littermates

\section{(Supplemental Figure 5).}


To understand individual microbial perturbations across RTT disease progression, we investigated the relationships between each ASV and Mecp2-e1 genotype in both male and female mice using limma voom ${ }^{47}$. Longitudinally, across disease progression, there was a similar number of ASVs that were associated with the main effect of genotype in both females (406) and males (433) (Figure 2a). Only 25\% (114) of genotype-associated ASVs in Mecp2-e1 $1^{-1+}$ females were also associated with Mecp2-e1 genotype in males, while 292 genotype-associated ASVs were identified in females versus 319 genotype-associated ASVs in Mecp2-e1-1y males only (Supplemental Tables 2 \& 3). Individual ASV and Mecp2-e1 genotype associations were significant as early as 5 weeks of age for both females and males, with the largest number of associations at 12 and 16 weeks in females and at 14 and 15 weeks in males (FDR<0.05; Figure $2 \mathbf{b}$ ). To identify dynamic changes in gut microbiota phyla across RTT disease progression, we identified ASVs with a significant genotype by age interaction, 591 in females compared with 585 in males (Supplemental

Table 4 \& 5). Visualization of the top 25 ASVs with genotype by age interactions within the fecal microbiota of Mecp2-e1 mutant mice compared to wild-type littermates across time, demonstrating the dynamic nature of the gut microbiome composition throughout disease progression (Figure 2c). Families representing the top 25 genotype by age ASVs in both sexes were Lachnospiraceae, Muribaculaceae, Ruminococcaceae, Streptococcaceae, and Burkholderiaceae, while those exclusive to females were Erysipelotrichaceae, Defluviitaleaceae, and Bifidobacteriaceae, and those exclusive to males were Lactobacillaceae, Family_XIII, Pseudomonadaceae, Staphylococcaceae, and Peptococcaceae. Together, this data demonstrate that the gut microbiome is dynamically altered during disease progression in both female and male Mecp2-e1 mutants, but that there are sexspecific signatures of specific families and ASVs.

\section{Fecal microbiota alterations in MeCP2e1 mutant mice precede and reflect longitudinal neurological, motor, and metabolic phenotypes}

To test the hypothesis that specific microbiota may impact RTT disease progression, each neurological, motor, and metabolic phenotype score was tested for association with each genotype-associated ASV. In females and males, 110 and 90 ASVs were associated with neurophenotyping score, 33 and 47 ASVs with gait, and 81 and 103 ASVs with body weight, respectively (Supplemental Tables 6-11). Figure 3 shows the RTT phenotype-associated ASVs that were persistently altered by genotype at multiple different time points throughout disease course, specifically those significantly associated with at least one phenotype, as well as with Mecp2-e1 genotype in at least $25 \%$ of the time points measured (four time points in females, three in males). Many ASVs associated with both body weight and genotype in females, including Rikenellaceae Alistipes, Lachnospiraceae NK4A136 group, Lachnospiraceae UCG-006, Ruminococcaceae Oscillibacter, Peptostreptococcaceae Romboutsia, and Ruminococcaceae Ruminococcus 1 genera (Figure 3a). Persistent genotype-related changes in these ASVs began concurrently with significant genotype-related body weight changes at 7 weeks of age. In contrast to Mecp2-e1 mutant females, males had far fewer ASVs that were associated with both body weight and genotype persistently (three compared to 10; Figure 3b). ASVs significantly associated with neurophenotyping score and genotype in females included Ruminococcaceae Candidatus Soleaferrea, Ruminococcaceae Oscillibacter, Peptostreptococcacea Romboutsia, and Lachnospiraceae Roseburia genera (Figure 3c). Remarkably, significant genotype-related changes in Romboutsia and Candidatus Soleaferrea were observed as early as 8 weeks, which was two weeks prior to significant neurophenotyping scores in Mecp2-e1 mutant females. In males, neurophenotype associated ASVs distinctly belonged to the genera Bifidobacteriaceae Bifidobacterium, Erysipelotrichaceae Faecalibaculum, and Ruminococcaceae Ruminiclostridium 9 (Figure 3d). Similar to females, Mecp2-e1 mutant males also demonstrated significant genotype-related differences in these ASVs prior to onset of neurophenotyping score deficits in mutant mice. We also observed a small number of ASVs associated with both gait and genotype persistently in females and males (two and one, respectively), and genotype-related changes in these ASVs were observed concurrently with onset of gait abnormalities in females but after onset of gait abnormalities in males (Supplemental Figure 6).

\section{Females exhibit stronger associations between fecal metabolites and Mecp2e1 genotype than males despite heterozygosity}

Since microbial communities in the gut produce a variety of key metabolites, such as short-chain fatty acids (SCFAs) relevant to nervous system metabolism, we tested the hypothesis that fecal metabolites may also precede disease phenotypes through longitudinal comparisons of Mecp2-e1 mutant and wild-type control littermates. We found that Mecp2-e $1^{-1+}$ female mutants, but not male mutants, had significantly different levels of fecal SCFAs across disease course compared to wild-type littermates (Figure 4a; Supplemental Figure 6). Specifically, female Mecp2-e $1^{-/+}$mutants had higher fecal levels of butyrate, isovalerate, and 
propionate than wild-type females (FDR<0.05), with genotype-related differences in butyrate levels that became evident as early as 5 weeks of age, and the most pronounced differences in SCFAs occurring at 9 weeks of age (Figure 4a; Supplemental Figure 6).

Striking sex-specific alterations were also identified through untargeted fecal metabolomics analysis of biogenic amines and lipids in mutant Mecp2-e1 mutant mice longitudinally. Mecp2-e1 genotype was associated with a larger number of both fecal biogenic amines and lipids longitudinally in Mecp2-e $1^{-1+}$ females compared to Mecp2-e $1^{-1 / y}$ males (Figure 4b, 4c). In females, 900 biogenic amines showed a main effect of Mecp2-e1 genotype, with 792 of those also showing an association with time and/or an interaction between genotype and time (FDR<0.05) (Figure 4b). Similar to biogenic amines, females also displayed stronger associations between Mecp2-e1 genotype and fecal lipids than males. In Mecp2-e $1^{-1+}$ females, 93 lipids were associated with genotype longitudinally, and of those, 66 were also associated with time and/or an interaction between genotype and time, further evidencing the dynamic relationship between Mecp2-e1 and the fecal metabolome across disease progression (Figure 4c).

Biogenic amines and lipids that could be accurately identified as known compounds and annotated to metabolite databases (89 and 169 , respectively) were used in enrichment testing using $\mathrm{ChemRICH}^{48}$ to identify clusters of related metabolites that were longitudinally associated with Mecp2-e1 genotype. There were 15 clusters of metabolites associated with genotype in Mecp2-

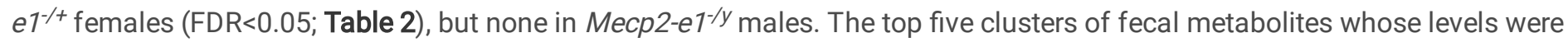

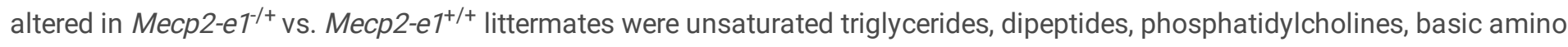
acids, and unsaturated fatty acids. Unsaturated triglycerides, indoles, unsaturated ceramides, and saturated triglycerides were primarily decreased in fecal matter collected longitudinally from Mecp2-e $1^{-/+}$compared to Mecp2-e $1^{+/+}$female littermates, whereas dipeptides, basic amino acids, azoles, phosphatidylethanolamines, pyridines, carnitines, amino acids, diglycerides, and saturated fatty acids were primarily increased. There was an equal split between the number of increased and decreased species of phosphatidylcholines and unsaturated fatty acids in female mutants compared to wild-type.

\section{Multi-variable associations of fecal metabolites with Mecp2e1 genotype, sex, age, phenotype, and microbiome}

To identify shifts in the fecal metabolome over disease course, we carried out a principal component analysis (PCA) and examined longitudinal patterns of metabolites that displayed a strong genotype by age interaction. In Mecp2-e $1^{-/+}$females, PCA plots for both biogenic amines and lipids showed that mutants clustered together with wild-type mice at 5 weeks of age, but separated from Mecp2-e $1^{+/+}$controls at 9 weeks and 19 weeks of age (Figure 5a, 5b). This indicates that the fecal metabolome

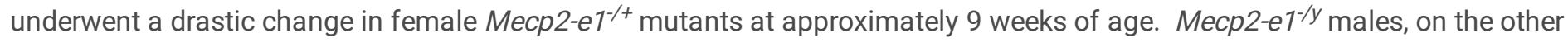
hand, did not show distinctive clustering by genotype or age in fecal biogenic amines, and showed clustering by genotype, but not age, in fecal lipidomic measures (Figure 5a, 5b). Examination of the top ten fecal biogenic amines and lipids with a significant genotype by age interaction further demonstrates that 9 weeks is a critical age at which the fecal metabolome in Mecp2-e $1^{-/+}$ female mutants diverged from wild-type Mecp2-e $1^{+/+}$littermates (Figure 5c, 5d). Furthermore, fecal lipids displayed a distinctive pattern in Mecp2-e $1^{-/ t}$ females indicating a relative increase of fecal lipids in mutants across disease progression. In contrast, the top ten biogenic amines and lipids with a genotype by age interaction identified in males showed differences in biogenic amines progressively throughout the disease course, whereas lipids displayed the largest differences at 5 weeks (Figure 5c, 5d). Notably, the biogenic amines with the most significant genotype by age interaction in both Mecp2-e1 mutant males and females were metabolites that were unable to be identified (unknowns). The most dynamic lipids across disease course were primarily long chain saturated and unsaturated fatty acids (LCFAs) and triglycerides in Mecp2-e $1^{-1+}$ females, but in Mecp2-e $1^{-/ y}$ males, they were primarily very long chain fatty acids (VLCFAs) and fatty acid esters of hydroxy fatty acids (FAHFAs). Together, these data demonstrate a female-specific dynamic shift in the fecal metabolome in Mecp2-e $1^{-/+}$mutant mice occurring as early as 5 weeks of age for butyrate and 9 weeks of age for lipids and biogenic amines.

In order to integrate the fecal metabolome with neurometabolic phenotypes and the microbiome, we applied a weighted gene coexpression network analysis (WGCNA) approach to metabolomic data. WGCNA reduced the complexity of individual metabolites into modules of highly correlated metabolites that were associated with genotype, age, fecal microbiota, and phenotypic traits. WGCNA analysis identified 18 fecal metabolite modules in females and 33 fecal metabolite modules in males (Supplemental Table 12 \& 13). Modules were assigned names by color, and metabolites that did not group into a network were placed into the 
grey module. Of the identified modules, five were associated with Mecp2-e1 genotype in females and six were associated with Mecp2-e1 genotype in males (Figure 6a, 6b). In females, genotype-associated metabolite modules were also highly related to age (three modules), fecal butyrate levels (two modules), gait as measured by overlap distance (three modules), body weight (three modules), and neuro-phenotyping score (three modules). Mecp2-e1 genotype-associated metabolite modules in males showed less significant correlation with these measures; only one of six modules was associated with gait and one was associated with neuro-phenotyping score, while there were none associated with age, fecal butyrate levels, or body weight. These data provide further evidence that fecal metabolites may be sensitive indicators of RTT progression, especially in females.

Mecp2-e1 genotype-associated fecal metabolite modules were also correlated with numerous fecal microbiota in both females and males. In females, Mecp2-e1 genotype-associated metabolite modules had a significant relationship with 28 ASVs (Figure 6c), and in males, Mecp2-e1 genotype-associated metabolite modules were associated with 56 ASVs (Figure 6d). Of these, 10 were also associated with gait, neuro-phenotyping score, and/or body weight in females, and 14 were also associated with at least one of these phenotypes in males, demonstrating a high degree of intersection between Mecp2-e1 genotype, the fecal microbiome, the fecal metabolome, and disease phenotype. The ASVs that were associated with genotype-related metabolite modules were from similar families across males and females, predominantly from the Lachnospiraceae and Ruminococcaceae families. Notably, one ASV, Ruminococcaceae Oscillibacter, was associated with two metabolite modules and all three disease phenotypes in females, and one ASV belonging to the Lachnospiraceae family of unknown genus was associated with two metabolite modules and all three disease phenotypes in males. The high degree of intersectionality between Mecp2-e1 genotype, the fecal microbiome, the fecal metabolome, and disease phenotypes suggest a significant role for the GI tract in RTT disease progression.

\section{Female mice deficient in MeCP2e1 have a distinctive brain lipid signature that reflects genotype-related alterations in fecal lipids}

The striking patterns of increased fecal lipids, including SCFAs, in Mecp2-e $1^{-/+}$females compared to controls across disease course indicate potential lipid malabsorption in the GI tract, which could impact the lipid composition of the brain. Furthermore, previous studies have found that lipid metabolism is disrupted in the brain of Mecp2 null mice 26,49,50. Thus, we tested the hypotheses that the lipidome was altered in the cortex from Mecp2-e1 mutants and correlated with the fecal metabolic changes. PCA plots of brain lipids supported this hypothesis and demonstrate a clear separation of Mecp2-e $1^{-1+}$ females from Mecp2-e $1^{+/+}$ littermates (Figure 7a). In contrast, males did not cluster separately by genotype for brain lipid composition. There were 35 lipids that differed by genotype in female brain at $p$-value $<0.05$, although these were no longer significant at FDR 0.05 (Supplemental

Table 14). Enrichment analysis of these lipids indicated that there were two clusters of down-regulated lipids in the cortex from mutant females compared to wild-type; one cluster of phosphatidylethanolamines and one of sphingomyelins (Table 3).

Unsaturated phosphatidylcholines also trended towards being depleted in Mecp2-e $1^{-/+}$females. In contrast, there were only five lipids altered in the cortex of Mecp2-e $1^{-/ y}$ compared to Mecp2-e1 $1^{+/ y}$ male littermates at $p<0.05$ (Supplemental Table 15), and there were no significantly enriched lipid clusters in males.

If lipid malabsorption in the GI tract influences lipid composition of the brain in RTT females, then fecal lipid levels should be associated with brain cortical lipids. In support of this hypothesis, we found a high degree of correlation between the top 10 genotype-associated fecal lipids at 9 weeks of age and the top 10 genotype-associated cortical lipids at 19 weeks of age in females (Figure 7b). These correlations were predominantly in the inverse direction, indicating that lipids that were increased in fecal matter were associated with decreased lipids in the brain cortices. Many of the lipids that were decreased in Mecp2-e $1^{-/+}$ cortex relative to Mecp2-e $1^{+/+}$female littermates are critical for neuronal function, including phosphatidylethanolamines, sphingomyelins, and phosphatidylcholines ${ }^{51-53}$. Thus, these data suggest that decreased absorption of lipids in the GI tract may negatively impact brain lipids in females with RTT.

\section{Discussion}

This study, the first of its kind, longitudinally characterized metabolite, microbial, and neuromotor profiles of RTT disease progression in a patient-relevant Mecp2-e1 mutant mouse model which provided critical insights into pathophysiology and new targets for potential therapies. We found a high degree of correlation between the gut microbiome, GI tract metabolism, and 
neuromotor phenotypes longitudinally across disease course, suggesting a role for the gut microbiome and metabolism in RTT disease progression, and uncovering critical disease-relevant microbiota and metabolites. Our findings revealed novel molecular signatures of gut microbial communities and metabolism that emerged prior to onset of neurological and motor phenotypes. Furthermore, this study uniquely identified the timing of molecular phenotypes in heterozygous Mecp2-e $1^{-/+}$females but not hemizygous Mecp2-e1-1y males, demonstrating that the intermediate phenotypes of RTT disease progression are sexually dimorphic (Figure 8). In females, Mecp2-e $1^{-/+}$mutants displayed alterations in fecal microbiota and metabolites as early as 5 weeks of age, prior to onset of disease phenotypes. The earliest disease phenotype that appeared in Mecp2-e $1^{-/+}$females was increased body weight at 7 weeks of age. At 9 weeks of age, Mecp2-e $1^{-/+}$females had a major shift in their fecal metabolome, just prior to the emergence of neurological and motor phenotypes at 10-11 weeks of age, and peak differences in the fecal microbiome at 12 weeks of age. In congruence with previous studies in RTT Mecp2 null mouse models, neuromotor phenotypes

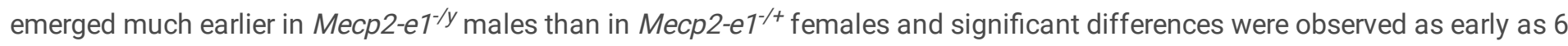
weeks of age, and early morbidity and mortality occurred relatively early in life at 16 weeks of age. Differences in fecal microbiota occurred concurrently with onset of gait and motor phenotypes in Mecp2-e1-1/ males and progressed throughout disease course. Although Mecp2-e $1^{-/ y}$ males trended towards an increased body weight compared to Mecp2-e1 $1^{+/ y}$ littermates, there were no statistically significant differences in body weight in male mice, in contrast to female mice. Furthermore, Mecp2-e1 $1^{-1 / y}$ males exhibited very few alterations in fecal metabolites and brain lipids compared to the more pronounced differences observed in Mecp2-e $1^{-1+}$ females. Taken together, our findings demonstrate a sex-specific RTT disease course and indicate that metabolic abnormalities in RTT precede symptom onset in females, suggesting a significant role of perturbed metabolism in disease pathophysiology and progression.

Two studies have previously demonstrated that female RTT patients have an altered gut microbial community compared to healthy controls 34,35 , but this is the first study to examine gut microbiota in a mouse model, which has the advantage of controlling diet and isolating the effect of mutations in Mecp2. Several of our findings were consistent with those in human RTT patients. Our finding in Mecp2-e $1^{-/+}$females that the reduced fecal abundance of Bacteroidetes was concurrent with an increase in Firmicutes was consistent with those of Strati et al. ${ }^{34}$ in human female patients with RTT, as well as in children with autism spectrum disorder (ASD) ${ }^{54}$ and in children with increased body mass index (BMI) ${ }^{55-57}$, suggesting a convergent profile reflective of humans with neurodevelopmental and metabolic dysfunction. Notably, Mecp2-e1-1/y mutant males showed the opposite results; Mecp2-e $1^{-/ y}$ mutant males had an increased relative abundance of Bacteroidetes and decreased relative abundance of Firmicutes, highlighting the sex-specific nature of RTT. Equivalent studies in RTT patients reported increased relative abundance of taxa belonging to the Clostridia class ${ }^{34,35}$, similar to children with ASD ${ }^{58}$, which was consistent with the female-specific increase in relative abundance of Clostridia longitudinally in Mecp2-e1 mutants compared to wild-type control littermates we observed. Several ASVs that were associated with both genotype, neurological phenotyping score, and body weight were from the Clostridia class, including Oscillibacter which was significantly and persistently increased in Mecp2-e $1^{-1+}$ females and also associated with neuro-phenotype, gait, and body weight. On the other hand, another member of the Clostridia class, Roseburia,

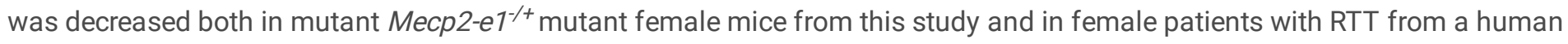
study ${ }^{35}$. We also found that an ASV belonging to the Roseburia genus was significantly associated with neurophenotype, though the difference in abundance of Roseburia did not emerge until five weeks after genotype-related differences in neurophenotype. Our results were also consistent with the two human RTT studies in the elevated levels several SCFAs in fecal samples, including propionate, butyrate, and isovalerate. Of note, propionate and isovalerate are produced by Clostridia. In contrast to female mice, male Mecp2-e $1^{-1 / y}$ mice did not exhibit consistencies with human RTT microbiome data, and in fact, showed some opposite effects. Mecp2-e1 $1^{-1 / y}$ male mice had decreased Actinobacteria, Erysipelotrichaceae, and Bifidobacterium, whereas they were increased in human RTT patients. There were also some inconsistencies between the fecal microbiome in female $M e c p 2-e 1^{-/+}$ mutants and human RTT patients. Most notably, Mecp2-e $1^{-/+}$mutant females had increased richness of the fecal microbiome, but female RTT patients had decreased alpha-diversity scores compared to healthy controls. Cumulatively, our findings experimentally confirmed the relationship between RTT and the gut microbiome from human RTT patients and further demonstrate that female mice are a better model of human RTT patients. In addition, our study allowed us to identify several taxa 
that were associated with disease phenotypes and Mecp2-e1 genotype that warrant further study in their potentially causative role in disease pathogenesis.

In recent years, metabolic dysfunction has been increasingly recognized as an important component of RTT disease pathology ${ }^{24}$. Our findings that female Mecp2-e1 mutant mice display progressive obesity and sex-specific alterations in the fecal metabolome corroborate this hypothesis. Importantly, we found that the fecal metabolome showed significant genotype-related differences prior to emergence of neurological and motor phenotypes, and that the fecal metabolome was also highly correlated with these phenotypes. Lipid metabolism, specifically, has been demonstrated to be dysregulated in both RTT patients and mouse models in previously published studies. Blood and plasma metabolites from RTT patients have indicated that cholesterol metabolism, sphingolipid metabolism, and fatty acid metabolism are perturbed in RTT ${ }^{49,59-63}$. Cholesterol and triglyceride metabolism has also been demonstrated to be dysregulated in the liver and brain in mouse models of RTT ${ }^{38,50,64-66}$. Our data support a role for lipid dysregulation in RTT. Mecp2-e1 mutant females, but not males, had altered fecal levels of many lipid species, including triglycerides, fatty acids, phosphatidylcholines, and phosphatidylethanolamines, beginning at 9 weeks of age, prior to onset of neuromotor phenotypes, suggesting that lipid dysregulation in the GI tract may play a role in disrupting neurological function. Further, the most dynamic genotype-associated fecal lipids were largely increased in female Mecp2-e1 mutants compared to wild-type littermates, which raises the possibility that deficient lipid absorption in the GI tract contributes to RTT pathology. Phosphatidylethanolamines, phosphatidylcholines, and sphingomyelins were also altered in the cortex of female mosaics, and these brain lipids were inversely associated with fecal lipid levels, providing additional evidence supporting this theory. Fecal and cortical lipids were predominantly influenced by Mecp2-e1 genotype in females, with few effects in males, indicating that the role of lipid dysregulation in the gut is specific to female disease progression in RTT.

Our findings demonstrated that RTT disease progression is sexually dimorphic and suggest that the underlying molecular pathology is inherently different in females than in males. This was surprising given that Mecp2-e $1^{-1+}$ mutant females have a wild-type copy Mecp2-e1 compared to Mecp2-e1-1/y males. X-linked dominant human disorders are quite rare, suggesting that the second copy of X-linked genes is usually protective. Female-specific effects in Mecp2-e1 mutant mice could be due to either sex hormones or cellular mosaicism. Sex hormones play a critical role in regulating metabolism ${ }^{67}$, and may therefore make females more susceptible to metabolic perturbations by deficits in MeCP2. However, cells that express mutant Mecp2 have been shown to have non-cell autonomous, or "bad neighborhood" effects, on MECP2 wild-type expressing cells in mosaic mutant females $15,16,68$. Specifically, these non-cell autonomous effects in astrocytes have been found to dampen dendritic arborization in mouse models ${ }^{16}$. Additional studies on sex hormonal and non-cell autonomous effects in metabolically active tissues such as the liver, adipose, and GI tract are needed to explore this potential mechanism for sex-specific effects in RTT.

The findings from this study provide insights into the underlying molecular pathways involved in RTT disease progression. Our data indicate that drugs that target specific gut microbiota or those that target lipid metabolism may be promising avenues for treatment of RTT. For example, Clostridia bacteria play a critical role in the gut-brain-axis ${ }^{46}$ and were increased in fecal samples from Mecp2-e $1^{-/+}$mutant females, so Clostridia-directed antibiotics may improve GI symptoms in girls with RTT. Omega-3 polyunsaturated fatty acids (PUFAs) are dietary fatty acids that are critical for proper neurodevelopment ${ }^{69}$ and have antiinflammatory effects ${ }^{70,71}$. Previous studies have shown partial rescue of RTT clinical symptoms with omega-3 PUFA supplementation ${ }^{72}$, but our study suggests potential lipid malabsorption in the GI tract. Thus, supplementation through other means, such as parenteral nutrition therapy, may have greater beneficial effects. Gene therapies are under development to treat RTT, but may take several years before they are widely available. Thus, repurposing existing FDA-approved therapeutics, such as antibiotics or omega-3 PUFA supplementation, could provide much needed relief for current RTT patients prior to and in addition to the availability of $M E C P 2$ gene therapies.

\section{Methods}

\section{Mouse Breeding and Cross Fostering}


Mecp2-e1 mutant and wild-type littermate controls were generated and maintained as described previously ${ }^{38,40}$. Briefly, Mecp2$e 1^{-/+}$mutant heterozygous females (Mecp2-e1 -/+) were bred with Mecp2-e1 wild-type C57BL/6J males (Jax 000664) to generate mutant heterozygous female offspring $\left(M e c p 2-e 1^{-/+}\right)$, mutant hemizygous male offspring (Mecp2-e $1^{-/ y}$, and wild-type female

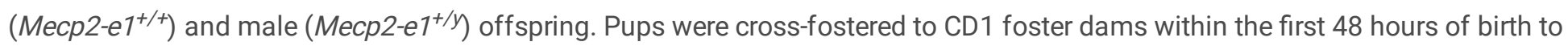
prevent the impact of poor maternal care from Mecp2-e $1^{-/+}$mutant dams. After weaning, mice were housed according to sex and genotype. Mice were maintained in a conventional temperature-controlled vivarium on a 12-hour light cycle with ad libitum access to food and water. All animal experiments were conducted in compliance with the National Institutes of Health Guidelines for the Care and use of Laboratory Animals and were carried out under approval and monitoring by the Institutional Animal Care and Use Committee (IACUC) of the University of California, Davis under IACUC protocol \#20621.

\section{Mouse Phenotyping}

Neuro-phenotyping and body weighing were performed weekly at three hours after the beginning of the light cycle (ZT3) as described previously ${ }^{40}$. Briefly, mice were evaluated weekly by the same researcher for ruffled coat, hypoactivity, open skin ulcers, abdomen size and specific responses to tail suspension including hind limb clasping, forelimb 'washing' and side to side flailing. Gait assessment was performed weekly between ZT3 and ZT5 as described previously ${ }^{38}$. Mice were habituated to paint application to feet on the day prior to gait analysis. The fore paws of mice were painted blue and the rear paws were painted red upon removal from home cage. Then mice were placed on blank paper strips in a chamber with a darkened distal end. The mouse was then allowed to walk down a straight alleyway lined with drawing paper. The resulting footprints were analyzed for stride length (distance between successive forelimb and successive hind limb prints), hind-base (distance between the right and left hind prints), front-base (distance between right and left front prints) and paw separation (distance between the forepaw and hind paw placement).

\section{Sample Collection}

Fecal pellets were collected weekly at three hours after the beginning of the light cycle (ZT3). Individual mice were placed in a clean cage for five minutes and accumulated fecal pellets were counted then collected, placed in tubes and frozen on dry ice prior to storage at $-80^{\circ} \mathrm{C}$. Cortex samples were collected at 3 hours after light cycle start after euthanasia via $\mathrm{CO}_{2}$ at 19 weeks of age for females and 16 weeks of age for males. Cortical samples were flash frozen in liquid nitrogen and stored at $-80^{\circ} \mathrm{C}$ prior to use.

\section{Characterization of the Fecal Microbiome}

Total microbial DNA was isolated from fecal pellets using Qiagen QIAmp PowerFecal DNA kit (Qiagen, Germantown, MD) and measured by spectrophotometry (Nanodrop, Thermo-Fisher, Waltham, MA). Primers 319F

(TCGTCGGCAGCGTCAGATGTGTATAAGAGACAG(spacer) GTACTCCTACGGGAGGCAGCAGT). and 806R (GTCTCGTGGGCTCGGAGATGTGTATAAGAGACAG(spacer) CCGGACTACNVGGGTWTCTAAT were used to amplify the V3-V4 domain of the 16S rRNA using a two-step PCR procedure. In step one of the amplification procedure, both forward and reverse primers contained an Illumina tag sequence (bold), a variable length spacer (no spacer, C, TC, or ATC for 319F; no spacer, G, TG, ATG for 806R) to increase diversity and improve the quality of the sequencing run, a linker sequence (italicized), and the $16 \mathrm{~S}$ target sequence (underlined). Each $25 \mathrm{ml} \mathrm{PCR}$ reaction contained 1 Unit Kapa2G Robust Hot Start Polymerase (Kapa Biosystems), $1.5 \mathrm{mM} \mathrm{MgCl}_{2}, 0.2 \mathrm{mM}$ final concentration dNTP mix, $0.2 \mathrm{mM}$ final concentration of each primer and 1ul of DNA for each sample. PCR conditions were: an initial incubation at $95^{\circ} \mathrm{C}$ for $3 \mathrm{~min}$, followed by 25 cycles of $95^{\circ} \mathrm{C}$ for $45 \mathrm{~s}, 50^{\circ} \mathrm{C}$ for $30 \mathrm{~s}$, $72^{\circ} \mathrm{C}$ for $30 \mathrm{~s}$ and a final extension of $72^{\circ} \mathrm{C}$ for $3 \mathrm{~min}$. In step two, each sample was barcoded with a unique forward and reverse barcode combination using forward primers (AATGATACGGCGACCACCGAGATCTACACNNNNNNNNTCGTCGGCAGCGTC) with an Illumina P5 adapter sequence (bold), a unique $8 \mathrm{nt}$ barcode (N), a partial matching sequence of the forward adapter used in step one (underlined), and reverse primers (CAAGCAGAAGACGGCATACGAGATNNNNNNNNGTCTCGTGGGCTCGG)) with an Illumina P7 adapter sequence (bold), unique $8 \mathrm{nt}$ barcode $(\mathrm{N})$, and a partial matching sequence of the reverse adapter used in step one (underlined). The PCR reaction in step two contained 1 Unit Kapa2G Robust Hot Start Polymerase (Kapa Biosystems), $1.5 \mathrm{mM}$ $\mathrm{MgCl}_{2}, 0.2 \mathrm{mM}$ final concentration dNTP mix, $0.2 \mathrm{mM}$ final concentration of each uniquely barcoded primer and $1 \mathrm{ul}$ of the 
product from the PCR reaction in step one diluted at a 10:1 ratio in water. PCR conditions were: an initial incubation at $95^{\circ} \mathrm{C}$ for 3 min, followed by 9 cycles of $95^{\circ} \mathrm{C}$ for $30 \mathrm{~s}, 58^{\circ} \mathrm{C}$ for $30 \mathrm{~s}, 72^{\circ} \mathrm{C}$ for $30 \mathrm{~s}$ and a final extension of $72^{\circ} \mathrm{C}$ for $3 \mathrm{~min}$.

The final product was quantified on the Qubit instrument using the Qubit Broad Range DNA kit (Invitrogen) and individual amplicons were pooled in equal concentrations. The pooled library was cleaned utilizing Ampure XP beads (Beckman Coulter) then the band of interest was further subjected to isolation via gel electrophoresis on a 1.5\% Blue Pippin HT gel (Sage Science). The library was quantified via qPCR followed by 300-bp paired-end sequencing using an Illumina MiSeq instrument in the Genome Center DNA Technologies Core, University of California, Davis.

Each sample was processed using a custom workflow designed to produce the highest quality amplicon sequence variants (ASVs) for each sample. Analysis of raw sequence reads began by first barcode demultiplexing to sample and 16S V3-V4 primer identification and trimming using dbcAmplicons (https://github.com/msettles/dbcAmplicons version 0.8.5). Any reads that could not be confidently assigned to sample by barcode, allowing one mismatch, or to primer sequences, allowing $\leq 4$ mismatches using the Levenshtein distance as long as the final 4 bases of the primer perfectly matched the target sequence, were discarded. The resulting forward and reverse reads were preprocessed using HTStream (https://github.com/s4hts/HTStream version 1.2.0) to overlap reads into full V3-V4 sequences while also excluding any reads that contained a no-call (' $\mathrm{N}$ ') character. Overlapped reads were then filtered, keeping amplicons $\geq 350 \mathrm{bp}$ in length, denoised, summarized to amplicon sequence variants (ASVs), and chimeric ASVs sequences removed using DADA2 (R version 3.6.1; DADA2 version 1.14.1) ${ }^{73}$. ASVs were then assigned to taxa using the RDP naïve Bayesian classifier ${ }^{74}$ implemented in DADA2 against the SILVA database reference of $16 \mathrm{~S}$ sequences $^{75,76}$. ASVs were aligned using DECIPHER and a phylogenetic tree of ASVs was constructed using phangorn ${ }^{77,78}$. Finally, rarefaction curves were calculated using the rarecurve function from the $\mathrm{R}$ package vegan.

Relative abundance of phyla and classes were calculated by normalizing read counts to library size using the relative log expression (RLE) normalization factor ${ }^{79}$ and then dividing normalized read counts in each phylum or class by the total number of normalized read counts in each sample to obtain a percentage of reads that belonged to each phyla and class. Analyses of differential abundance of individual ASVs was carried out using limma voom ${ }^{47}$ in Bioconductor (www.bioconductor.org) modeling the effect of Mecp2-e1 genotype longitudinally across disease course, stratified by sex and controlling for within-litter effects. This analysis generated overall longitudinal associations between genotype and individual ASVs, as well as associations between ASVs and genotype cross-sectionally at each time point. The Benjamini-Hochberg method was used to correct for multiple comparisons and obtain false discovery rates (FDRs). FDR values of less than 0.05 were considered statistically significant.

\section{Characterization of the Fecal and Cortical Metabolome}

Fecal samples collected at 5, 9, and 19 weeks from females and 5, 9, and 16 weeks from males were processed and assessed for short chain fatty acids (SCFAs), biogenic amines and lipids, and cortical samples were processed and assessed for lipids at the West Coast Metabolomics Center (WCMC) at UC Davis. For SCFA analysis, fecal samples were processed by homogenization of $10 \mathrm{mg}$ of fecal matter followed by solvent extraction using HPLC-grade ethylbutyric acid, hydrochloric acid, tert-butyl methyl ether (MTBE), and methylvaleric acid. SCFAs were measured using gas chromatography coupled with mass spectrometry (GCMS) on the Agilent GC7890B/5977MS with DB5 Duraguard 30m x 0.25mm x 0.25 u capillary column and measured against standards of known concentrations ${ }^{80,81}$. To analyze biogenic amines and lipids, samples were processed via homogenization followed by extraction with a bi-phasic solvent system comprised of methanol, MTBE, and water, which allowed for extraction of biogenic amines in the aqueous phase and lipids in the lipid phase ${ }^{82}$. To measure biogenic amines, polar phase lipid extraction by ultra high pressure liquid chromatography (UHPLC) was used coupled with mass spectrometer (MS). UHPLC was performed using an Agilent Infinity LC system with a Waters BEH Amide Column, and MS was carried out using a SCIEX Triple time of flight (TOF) 6600 mass spectrometer. To measure lipids, UHPLC was carried out on an Agilent 1290 Infinity LC system with a Waters CSH C18 column interfaced to a quadrupole TOF MS. Data were processed using an untargeted approach by first using mzMine 2.0 software to detect peaks and then collated with Agilent's MassHunter quantification method using retention time and exact mass, and the NIST14 / Metlin / MassBank / Lipidblast libraries ${ }^{83-85}$ to identify metabolites with manual confirmation of adduct ions and spectral scoring accuracy. To quantify data, peak heights were normalized using vector normalization to the sum of all peak

Page $11 / 27$ 
heights for identified metabolites. Lipids underwent further quantification to obtain semi-quantitative data for known lipids. Quality control (QC) samples were included with each analysis.

Metaboanalyst (metaboanalyst.ca) ${ }^{86}$ was used to further process data and carry out statistical analyses to compare fecal metabolites across genotype and stratified by sex, longitudinally across time, and to compare cortical lipids across genotype cross-sectionally at 19 weeks for females and 16 weeks for males. Prior to statistical analysis, data underwent filtering to remove metabolites that were higher than $25 \%$ relative standard deviation (standard deviation/mean) in QC samples and to remove metabolites that were nearly constant across the experiment and were within the interquantile range (IQR). Metabolites that passed filtering were then log transformed. For fecal metabolites, repeated measures two-way ANOVA was carried out to compare mutant vs. wild-type females and males longitudinally, and for cortical lipids, a t-test was carried out. FDRs were calculated using the Benjamini-Hochberg method for each metabolite/lipid. An FDR $<0.05$ was considered statistically significant for fecal metabolites, but a raw p-value threshold of 0.05 was used for cortical lipids. This was because we observed more subtle effects of Mecp2-e1 genotype on lipids in the cortex than in fecal matter.

\section{Metabolomics Enrichment Analysis}

Metabolite enrichment analyses were carried out using $\mathrm{ChemRICH}^{48}$, which utilizes chemical ontologies and chemical structures to cluster metabolites in a study-specific manner to yield enriched groups of metabolites. Biogenic amines and lipids that were annotated to specific compounds were then further annotated with InChIKeys, PubchemIDs, and SMILES using the Chemical Translation Service (http://cts.fiehnlab.ucdavis.edu/) and the PubChem Identifier Exchange Tool (https://pubchem.ncbi.nlm.nih.gov/idexchange/idexchange.cgi). Compounds that were able to be fully annotated and their genotype-associated FDR values and foldchange in mutant vs. wild-type mice were used for ChemRICH enrichment testing. Raw p-values were used in lieu of FDR values for cortical lipids since brain lipids showed more subtle differences. We considered clusters to be significantly enriched if FDR $<0.05$ for fecal metabolomics and FDR $<0.10$ for cortical lipids.

\section{Weighted Gene Co-Expression Network Analysis (WGCNA) for Fecal Metabolomics}

To condense fecal metabolomics data into groups of co-regulated metabolites, we employed a Weighted Gene Co-Expression Network Analysis (WGCNA) to processed fecal metabolomics data. WGCNA analysis is commonly carried out to identify coregulated gene networks using microarray and RNA-seq data ${ }^{87}$, and here we applied it to metabolomics data to obtain modules of metabolite networks. Data were stratified by sex for the analysis. We selected a soft power threshold based on scale independence where the model fit $\left(R^{2}\right)$ was 0.8 , which was 18 for females and 5 for males (Supplemental Figure 9). The minimum number of metabolites allowed in each network module was 10. The resulting network analysis resulted in 19 fecal metabolite modules for females and 33 for males (Supplemental Figure 10). These resulting modules containing metabolites that were highly correlated with one another were then used in data integration to identify relationships between fecal metabolites, the fecal microbiome, and disease phenotypes.

\section{Statistical Analysis and Data Integration}

All statistical analyses were carried out using R version 3.6.3 (www.r-project.org). To compare differences in phenotypic measurements between mutant and wild-type mice, we utilized linear mixed effects models for longitudinal data to control for repeated measures and with a random effect variable to control for within-litter effects. In addition to longitudinal analyses, we also carried out cross-sectional analyses at each time point and then used the Benjamini-Hochberg method to calculate FDRs and adjust for multiple comparisons across each time point. All analyses were stratified by sex in order to detect sex-specific effects of Mecp2-e1 genotype.

To integrate microbiome data with phenotypic data, limma voom was used to test the effect of phenotype on ASVs, while controlling for genotype, age, and within-litter effects. To integrate metabolomics data with phenotypes, genotype, and age, linear mixed effects models were used to model the effect of each of these variables on the EigenValue for each metabolome module as calculated via WGCNA. These models contained random effects variables to correct for within-litter effects and repeated measures. Microbiome and metabolomics data was integrated by using limma voom to model the effect of metabolomic module 
EigenValues on ASVs while adjusting for genotype, age, and within-litter effects. To evaluate the relationship between fecal lipids and cortical lipids, Spearman's correlation coefficients were calculated. All analyses were corrected for multiple comparisons using the Benjamini-Hochberg method to calculate FDRs.

\section{Declarations}

\section{ACKNOWLEDGEMENTS}

This work was funded by NIH R01 AA027075 to JML and the UC Davis Intellectual and Developmental Disabilities Research Center (IDDRC) [P50HD103526]. The sequencing was carried out by the DNA Technologies and Expression Analysis Cores at the UC Davis Genome Center and was supported by a NIH Shared Instrumentation Grant [1S100D010786-01].

\section{AUTHOR CONTRIBUTIONS}

KN participated in study design, analyzed and interpreted the data, and drafted the manuscript. JML conceptualized and designed the study, obtained funding, aided in interpretation of data, and revised the manuscript. DHY conceptualized and designed the study, acquired data, assisted in data interpretation, and revised the manuscript. BDJ, MLS, and SSH participated in data analysis. TEG, RLP, DC, SMH, KMY, and MR acquired data.

\section{COMPETING INTERESTS STATEMENT}

The authors declare no competing interests.

\section{References}

1. Jeffrey L. Neul, J. L. et al. Rett syndrome: Revised diagnostic criteria and nomenclature. Ann. Neurol. 68, 944-950 (2010).

2. Amir, R. E. et al. Rett syndrome is caused by mutations in X-linked MECP2, encoding methyl- CpG-binding protein 2. Nat. Genet. 23, 185-188 (1999).

3. Meehan, R., Lewis, J. D. \& Bird, A. P. Characterization of MECP2, a vertebrate DNA binding protein with affinity for methylated DNA. Nucleic Acids Res. 20, 5085-5092 (1992).

4. Mellén, M., Ayata, P., Dewell, S., Kriaucionis, S. \& Heintz, N. MeCP2 binds to 5hmC enriched within active genes and accessible chromatin in the nervous system. Cel/ 151, 1417-1430 (2012).

5. Guo, J. U. et al. Distribution, recognition and regulation of non-CpG methylation in the adult mammalian brain. Nat. Neurosci. 17, 215-222 (2014).

6. Gabel, H. W. et al. Disruption of DNA-methylation-dependent long gene repression in Rett syndrome. Nature 522, 89-93 (2015).

7. Lavery, L. A. et al. Losing dnmt3a dependent methylation in inhibitory neurons impairs neural function by a mechanism impacting rett syndrome. Elife 9 , (2020).

8. Buchmuller, B. C., Kosel, B. \& Summerer, D. Complete Profiling of Methyl-CpG-Binding Domains for Combinations of Cytosine Modifications at CpG Dinucleotides Reveals Differential Read-out in Normal and Rett-Associated States. Sci. Rep. 10, (2020).

9. Nan, X., Campoy, F. J. \& Bird, A. MeCP2 is a transcriptional repressor with abundant binding sites in genomic chromatin. Cell 88, 471-481 (1997).

10. Chahrour, M. et al. MeCP2, a key contributor to neurological disease, activates and represses transcription. Science (80-.) ). 320, 1224-1229 (2008).

11. Szulwach, K. E. et al. Cross talk between microRNA and epigenetic regulation in adult neurogenesis. J. Cell Biol. 189, 127141 (2010).

12. Khan, A. W. et al. MeCP2 interacts with chromosomal microRNAs in brain. Epigenetics 12, 1028-1037 (2017).

13. Young, J. I. et al. Regulation of RNA splicing by the methylation-dependent transcriptional repressor methyl-CpG binding protein 2. Proc. Natl. Acad. Sci. U. S. A. 102, 17551-17558 (2005). 
14. Georgel, P. T. et al. Chromatin compaction by human MeCP2. Assembly of novel secondary chromatin structures in the absence of DNA methylation. J. Biol. Chem. 278, 32181-32188 (2003).

15. Renthal, W. et al. Characterization of human mosaic Rett syndrome brain tissue by single-nucleus RNA sequencing. Nat. Neurosci. 21, 1670-1679 (2018).

16. Maezawa, I., Swanberg, S., Harvey, D., LaSalle, J. M. \& Jin, L. W. Rett syndrome astrocytes are abnormal and spread MeCP2 deficiency through gap junctions. J. Neurosci. 29, 5051-5061 (2009).

17. Braunschweig, D., Simcox, T., Samaco, R. C. \& LaSalle, J. M. X-chromosome inactivation ratios affect wild-type MeCP2 expression within mosaic Rett syndrome and Mecp2-/+ mouse brain. Hum. Mol. Genet. 13, 1275-1286 (2004).

18. Trappe, R. et al. MECP2 mutations in sporadic cases of Rett syndrome are almost exclusively of paternal origin. Am. J. Hum. Genet. 68, 1093-1101 (2001).

19. Matagne, V. et al. Severe offtarget effects following intravenous delivery of AAV9-MECP2 in a female mouse model of Rett syndrome. Neurobiol. Dis. 149, (2021).

20. Hagberg, B., Aicardi, J., Dias, K. \& Ramos, O. A progressive syndrome of autism, dementia, ataxia, and loss of purposeful hand use in girls: Rett's syndrome: Report of 35 cases. Ann. Neurol. 14, 471-479 (1983).

21. Bienvenu, T. et al. MECP2 mutations account for most cases of typical forms of Rett syndrome. Hum. Mol. Genet. 9, 13771384 (2000).

22. Symons, F. J., Byiers, B., Tervo, R. C. \& Beisang, A. Parent-reported pain in Rett syndrome. Clin. J. Pain 29, 744-746 (2013).

23. Peron, A. et al. Phenotypes in adult patients with Rett syndrome: Results of a 13-year experience and insights into healthcare transition. J. Med. Genet. 0, 1-7 (2020).

24. Justice, M., Buchovecky, C., Kyle, S. \& Djukic, A. A role for metabolism in Rett syndrome pathogenesis. Rare Dis. 18, (2013).

25. Millar-Büchner, P. et al. Severe changes in colon epithelium in the Mecp2-null mouse model of Rett syndrome. Mol. Cell. Pediatr. 3, 37 (2016).

26. Buchovecky, C. M. et al. A suppressor screen in Mecp2 mutant mice implicates cholesterol metabolism in Rett syndrome. Nat. Genet. 45, 1013-1020 (2013).

27. Maslowski, K. M. \& MacKay, C. R. Diet, gut microbiota and immune responses. Nature Immunology vol. 12 5-9 (2011).

28. Zheng, P. et al. The gut microbiome from patients with schizophrenia modulates the glutamate-glutamine-GABA cycle and schizophrenia-relevant behaviors in mice. Sci. Adv. 5, (2019).

29. Yang, J. et al. Landscapes of bacterial and metabolic signatures and their interaction in major depressive disorders. Sci. Adv. 6. (2020).

30. Baldini, F. et al. Parkinson's disease-associated alterations of the gut microbiome predict disease-relevant changes in metabolic functions. BMC Biol. 18, (2020).

31. Mayer, E. A., Padua, D. \& Tillisch, K. Altered brain-gut axis in autism: Comorbidity or causative mechanisms? BioEssays $\mathbf{3 6}$, 933-939 (2014).

32. Sampson, T. R. \& Mazmanian, S. K. Control of brain development, function, and behavior by the microbiome. Cell Host and Microbe vol. 17 565-576 (2015).

33. Wang, Y. \& Kasper, L. H. The role of microbiome in central nervous system disorders. Brain, Behavior, and Immunity vol. 38 112 (2014).

34. Strati, F. et al. Altered gut microbiota in Rett syndrome. Microbiome 4, 41 (2016).

35. Borghi, E. et al. Rett Syndrome: A Focus on Gut Microbiota. Int. J. Mol. Sci. 18, 344 (2017).

36. Hagberg, B. \& Witt-Engerstrom, I. Rett syndrome: A suggested staging system for describing impairment profile with increasing age towards adolescence. Am. J. Med. Genet. 24, 47-59 (1986).

37. Cianfaglione, R. et al. Ageing in Rett syndrome. J. Intellect. Disabil. Res. 60, 182-190 (2016).

38. Vogel Ciernia, A. et al. MeCP2 isoform e1 mutant mice recapitulate motor and metabolic phenotypes of Rett syndrome. Hum. Mol. Genet. 27, 4077-4093 (2018). 
39. Vogel Ciernia, A. et al. Early motor phenotype detection in a female mouse model of Rett syndrome is improved by crossfostering. Hum. Mol. Genet. 26, 1839-1854 (2017).

40. Yasui, D. et al. Mice with an isoform-ablating Mecp2 exon 1 mutation recapitulate the neurologic deficits of Rett syndrome. Hum. Mol. Genet. 23, 2447-2458 (2014).

41. El-Khoury, R. et al. GABA and glutamate pathways are spatially and developmentally affected in the brain of Mecp2-deficient mice. PLoS One 9 , (2014).

42. Wang, I. T. J., Reyes, A. R. S. \& Zhou, Z. Neuronal morphology in MeCP2 mouse models is intrinsically variable and depends on age, cell type, and Mecp2 mutation. Neurobiol. Dis. 58, 3-12 (2013).

43. Olson, C. O., Zachariah, R. M., Ezeonwuka, C. D., Liyanage, V. R. B. \& Rastegar, M. Brain region-specific expression of MeCP2 isoforms correlates with DNA methylation within Mecp2 regulatory elements. PLoS One 9, (2014).

44. Guy, J., Hendrich, B., Holmes, M., Martin, J. E. \& Bird, A. A mouse Mecp2-null mutation causes neurological symptoms that mimic rett syndrome. Nat. Genet. 27, 322-326 (2001).

45. Maurice, C. F. et al. Marked seasonal variation in the wild mouse gut microbiota. ISME J. 9, 2423-2434 (2015).

46. Labus, J. S. et al. Evidence for an association of gut microbial Clostridia with brain functional connectivity and gastrointestinal sensorimotor function in patients with irritable bowel syndrome, based on tripartite network analysis. Microbiome 7, (2019).

47. Law, C. W., Chen, Y., Shi, W. \& Smyth, G. K. Voom: Precision weights unlock linear model analysis tools for RNA-seq read counts. Genome Biol. 15, (2014).

48. Barupal, D. K. \& Fiehn, O. Chemical Similarity Enrichment Analysis (ChemRICH) as alternative to biochemical pathway mapping for metabolomic datasets. Sci. Rep. 7, (2017).

49. Segatto, M. et al. Cholesterol Metabolism Is Altered in Rett Syndrome: A Study on Plasma and Primary Cultured Fibroblasts Derived from Patients. PLoS One 9, e104834 (2014).

50. Lütjohann, D., Lopez, A. M., Chuang, J.-C., Kerksiek, A. \& Turley, S. D. Identification of Correlative Shifts in Indices of Brain Cholesterol Metabolism in the C57BL6/ Mecp2 ${ }^{\text {tm1.1Bird }}$ Mouse, a Model for Rett Syndrome. Lipids 53, 363-373 (2018).

51. Paoletti, L., Elena, C., Domizi, P. \& Banchio, C. Role of Phosphatidylcholine during Neuronal differentiation. IUBMB Life vol. 63 714-720 (2011).

52. Schneider, N. et al. Sphingomyelin in brain and cognitive development: Preliminary data. eNeuro 6, (2019).

53. Vance, J. E. \& Tasseva, G. Formation and function of phosphatidylserine and phosphatidylethanolamine in mammalian cells. Biochimica et Biophysica Acta - Molecular and Cell Biology of Lipids vol. 1831 543-554 (2013).

54. Strati, F. et al. New evidences on the altered gut microbiota in autism spectrum disorders. Microbiome 5, (2017).

55. Riva, A. et al. Pediatric obesity is associated with an altered gut microbiota and discordant shifts in Firmicutes populations. Environ. Microbiol. 19, 95-105 (2017).

56. Indiani, C. M. D. S. P. et al. Childhood Obesity and Firmicutes/Bacteroidetes Ratio in the Gut Microbiota: A Systematic Review. Childhood Obesity vol. 14 501-509 (2018).

57. Fernandes, J., Su, W., Rahat-Rozenbloom, S., Wolever, T. M. S. \& Comelli, E. M. Adiposity, gut microbiota and faecal short chain fatty acids are linked in adult humans. Nutr. Diabetes 4, (2014).

58. Finegold, S. M. et al. Gastrointestinal Microflora Studies in Late-Onset Autism. Clin. Infect. Dis. 35, S6-S16 (2002).

59. Cappuccio, G. et al. Sphingolipid metabolism perturbations in rett syndrome. Metabolites 9, (2019).

60. Sticozzi, C. et al. Scavenger receptor B1 post-translational modifications in Rett syndrome. FEBS Lett. 587, 2199-2204 (2013).

61. Signorini, C. et al. F4-neuroprostanes mediate neurological severity in Rett syndrome. Clin. Chim. Acta 412, 1399-1406 (2011).

62. Leoncini, S. et al. Oxidative stress in Rett syndrome: Natural history, genotype, and variants. Redox Rep. 16, 145-153 (2011).

63. De Felice, C. et al. F2-dihomo-isoprostanes as potential early biomarkers of lipid oxidative damage in Rett syndrome. J. Lipid Res. 52, 2287-2297 (2011). 
64. Buchovecky, C. M. et al. A suppressor screen in Mecp2 mutant mice implicates cholesterol metabolism in Rett syndrome. Nat. Genet. 45, 1013-1020 (2013).

65. Lopez, A. M., Chuang, J. C., Posey, K. S. \& Turley, S. D. Suppression of brain cholesterol synthesis in male Mecp2-deficient mice is age dependent and not accompanied by a concurrent change in the rate of fatty acid synthesis. Brain Res. 1654, 7784 (2017).

66. Pacheco, N. L. et al. RNA sequencing and proteomics approaches reveal novel deficits in the cortex of Mecp2-deficient mice, a model for Rett syndrome. Mol. Autism 8, 56 (2017).

67. Comitato, R., Saba, A., Turrini, A., Arganini, C. \& Virgili, F. Sex Hormones and Macronutrient Metabolism. Critical Reviews in Food Science and Nutrition vol. 55 227-241 (2015).

68. Yasui, D. H. et al. MeCP2 modulates gene expression pathways in astrocytes. Mol. Autism 4, 3 (2013).

69. Weiser, M. J., Butt, C. M. \& Mohajeri, M. H. Docosahexaenoic acid and cognition throughout the lifespan. Nutrients vol. 8 (2016).

70. De Caterina, R., Liao, J. K. \& Libby, P. Fatty acid modulation of endothelial activation. in American Journal of Clinical Nutrition vol. 71 (American Society for Nutrition, 2000).

71. Mas, E. et al. The omega-3 fatty acids EPA and DHA decrease plasma F2- isoprostanes: Results from two placebo-controlled interventions. Free Radic. Res. 44, 983-990 (2010).

72. De Felice, C. et al. Partial rescue of Rett syndrome by $\omega-3$ polyunsaturated fatty acids (PUFAs) oil. Genes Nutr. 7, 447-458 (2012).

73. Callahan, B. J. et al. DADA2: High-resolution sample inference from Illumina amplicon data. Nat. Methods 13, 581-583 (2016).

74. Wang, Q., Garrity, G. M., Tiedje, J. M. \& Cole, J. R. Naïve Bayesian classifier for rapid assignment of rRNA sequences into the new bacterial taxonomy. Appl. Environ. Microbiol. 73, 5261-5267 (2007).

75. Yilmaz, P. et al. The SILVA and 'all-species Living Tree Project (LTP)' taxonomic frameworks. Nucleic Acids Res. 42, (2014).

76. Quast, C. et al. The SILVA ribosomal RNA gene database project: Improved data processing and web-based tools. Nucleic Acids Res. 41, (2013).

77. Wright, E. S. Using DECIPHER v2.0 to analyze big biological sequence data in R. $R$ J. 8, 352-359 (2016).

78. Schliep, K. P. phangorn: Phylogenetic analysis in R. Bioinformatics 27, 592-593 (2011).

79. Anders, S. \& Huber, W. Differential expression analysis for sequence count data. Genome Biol. 11, (2010).

80. Richardson, A. J., Calder, A. G., Stewart, C. S. \& Smith, A. Simultaneous determination of volatile and non-volatile acidic fermentation products of anaerobes by capillary gas chromatography. Lett. Appl. Microbiol. 9, 5-8 (1989).

81. Moreau, N. M. et al. Simultaneous measurement of plasma concentrations and 13C-enrichment of short-chain fatty acids, lactic acid and ketone bodies by gas chromatography coupled to mass spectrometry. J. Chromatogr. B Anal. Technol. Biomed. Life Sci. 784, 395-403 (2003).

82. Matyash, V., Liebisch, G., Kurzchalia, T. V., Shevchenko, A. \& Schwudke, D. Lipid extraction by methyl-terf-butyl ether for highthroughput lipidomics. in Journal of Lipid Research vol. 49 1137-1146 (J Lipid Res, 2008).

83. Xue, J., Guijas, C., Benton, H. P., Warth, B. \& Siuzdak, G. METLIN MS2 molecular standards database: a broad chemical and biological resource. Nature Methods vol. 17 953-954 (2020).

84. Horai, H. et al. MassBank: A public repository for sharing mass spectral data for life sciences. J. Mass Spectrom. 45, 703714 (2010).

85. Kind, T. et al. LipidBlast in silico tandem mass spectrometry database for lipid identification. Nat. Methods 10, 755-758 (2013).

86. Pang, Z., Chong, J., Li, S. \& Xia, J. Metaboanalystr 3.0: Toward an optimized workflow for global metabolomics. Metabolites 10, (2020).

87. Zhang, B. \& Horvath, S. A general framework for weighted gene co-expression network analysis. Stat. Appl. Genet. Mol. Biol. 4, (2005). 


\section{Tables}

Table 1. Longitudinal analyses of relative phyla abundance and diversity scores by Genotype

\begin{tabular}{|lllll|}
\hline & Females & \multicolumn{3}{l|}{ Males } \\
\hline Phylum & Beta (\%) & p-value & Beta (\%) & p-value \\
\hline Firmicutes & $\mathbf{3 . 7 2 0 0 0}$ & $\mathbf{0 . 0 3 3}$ & $-\mathbf{5 . 1 3 0 0 0}$ & $\mathbf{0 . 0 1 0}$ \\
\hline Actinobacteria & -0.02950 & $\mathbf{0 . 2 6}$ & $-\mathbf{0 . 1 8 0 0}$ & $\mathbf{0 . 0 0 0 0 7 6}$ \\
\hline Cyanobacteria & -0.00022 & 0.78 & 0.000001 & 0.99 \\
\hline Fusobacteria & -0.00011 & 0.82 & -0.00035 & 0.48 \\
\hline Patescibacteria & 0.00054 & 0.94 & -0.013 & 0.12 \\
\hline Proteobacteria & -0.08200 & 0.53 & -0.2200 & 0.46 \\
\hline Tenericutes & 0.04600 & 0.72 & $\mathbf{0 . 3 0 0 0}$ & $\mathbf{0 . 0 3 1}$ \\
\hline Verrucomicrobia & $\mathbf{- 0 . 2 8 0 0 0}$ & $\mathbf{0 . 0 2 3}$ & 0.00385 & 0.17 \\
\hline Diversity Index & Beta & $\mathrm{p}$-value & Beta & $\mathrm{p}$-value \\
\hline Shannon & $\mathbf{0 . 1 2 4}$ & $\mathbf{0 . 0 4}$ & 0.027 & 0.59 \\
\hline Chao & $\mathbf{4 8 . 2}$ & $\mathbf{0 . 0 1 3}$ & -30.6 & 0.097 \\
\hline
\end{tabular}

Relative abundance (\%) and diversity indices were compared longitudinally across genotypes using linear mixed effects models and stratified by sex. Betas are the mean difference in genotypes longitudinally across disease course (between 5 and 19 weeks for females and 5 and 16 weeks for males). Phyla and their respected values are bolded where $p<0.05$. N=11-19/genotype/sex.

Table 2. Fecal metabolite clusters significantly altered (FDR<0.05) in Mecp2e1 -/+ vs. Mecp2e1 +/+ females 


\begin{tabular}{|c|c|c|c|c|c|c|c|}
\hline Cluster name & $\begin{array}{l}\text { Cluster } \\
\text { size }\end{array}$ & p-value & FDR & Key compound & $\begin{array}{l}\text { Altered } \\
\text { metabolites }\end{array}$ & Increased & Decreased \\
\hline Unsaturated triglycerides & 39 & $1.5 \mathrm{E}-16$ & 3.7E-15 & TAG 52:1 & 26 & 8 & 18 \\
\hline Dipeptides & 4 & $2.9 \mathrm{E}-08$ & 2.3E-07 & Ala-Ala & 4 & 3 & 1 \\
\hline Phosphatidylcholines & 26 & $3.4 \mathrm{E}-08$ & 2.3E-07 & PC 32:0 & 10 & 5 & 5 \\
\hline Amino acids, basic & 5 & $3.8 \mathrm{E}-08$ & 2.3E-07 & N-Methyltyrosine & 5 & 4 & 1 \\
\hline Unsaturated fatty acids & 24 & 0.000036 & 0.00017 & FA 18:2 & 10 & 5 & 5 \\
\hline Indoles & 3 & 0.0001 & 0.0004 & $\begin{array}{l}\mathrm{N} \text {-alpha.-Acetyl- } \\
\mathrm{L} \text {-arginine }\end{array}$ & 3 & 1 & 2 \\
\hline Azoles & 3 & 0.00015 & 0.0005 & Creatinine & 2 & 2 & 0 \\
\hline Phosphatidylethanolamines & 8 & 0.00034 & 0.001 & PE 34:1 & 3 & 2 & 1 \\
\hline Pyridines & 8 & 0.00084 & 0.0022 & 3-Aminopyridine & 4 & 4 & 0 \\
\hline Carnitines & 5 & 0.0013 & 0.0031 & $\begin{array}{l}\text { 3- } \\
\text { Dehydrocarnitine }\end{array}$ & 3 & 3 & 0 \\
\hline Unsaturated ceramides & 8 & 0.0023 & 0.005 & Ceramide d39:1 & 5 & 1 & 4 \\
\hline Saturated triglycerides & 3 & 0.0025 & 0.005 & TAG 46:0 & 3 & 0 & 3 \\
\hline Amino acids & 5 & 0.0047 & 0.0087 & Isoleucine & 3 & 3 & 0 \\
\hline Diglycerides & 8 & 0.021 & 0.037 & DG 38:5 & 4 & 3 & 1 \\
\hline Saturated FA & 18 & 0.031 & 0.05 & FA 20:0 & 3 & 3 & 0 \\
\hline
\end{tabular}

Chemical enrichment clustering analysis was carried out using CHEMRich to identify clusters of chemically similar fecal metabolites that were significantly altered in mutant $(-/+)$ vs. wild-type $(+/+)$ females longitudinally across disease course (measurements taken at 5,9 , and 19 weeks of age). Cluster size is the number of metabolites in each cluster and altered metabolites are the number of metabolites within that cluster that were altered in mutant vs. wild-type females. The number of altered metabolites that were increased and decreased in mutant vs. wild-type females are represented in the adjacent columns. $\mathrm{N}=8 /$ genotype.

Table 3. Brain lipid clusters significantly altered (FDR<0.10) in Mecp2e1 -/+ vs. Mecp2e1 +/+ females

\begin{tabular}{|lllllllc|}
\hline Cluster name & $\begin{array}{l}\text { Cluster } \\
\text { size }\end{array}$ & p-value & FDR & Key compound & $\begin{array}{l}\text { Altered } \\
\text { metabolites }\end{array}$ & Increased & Decreased \\
\hline NewCluster_11 & 4 & 0.00094 & 0.013 & SM d40:0 & 2 & 0 & 2 \\
\hline NewCluster_6 & 9 & 0.004 & 0.028 & $\begin{array}{l}\text { PE p-34:1/PE o- } \\
34: 2\end{array}$ & 3 & 0 & 3 \\
\hline $\begin{array}{l}\text { Unsaturated } \\
\text { phosphatidylcholines }\end{array}$ & 36 & 0.015 & 0.068 & $\begin{array}{l}\text { PC p-36:3; or PC } \\
\text {--36:4 }\end{array}$ & 5 & 2 & 3 \\
\hline
\end{tabular}

Chemical enrichment clustering analysis was carried out using CHEMRich to identify clusters of chemically similar cortical metabolites that were significantly altered in mutant $(-/+)$ vs. wild-type $(+/+)$ females at 19 weeks of age. Cluster size is the number of metabolites in each cluster and altered metabolites are the number of metabolites within that cluster that were altered in mutant vs. wild-type females. The number of altered metabolites that were increased and decreased in mutant vs. wild-type females are represented in the adjacent columns. $\mathrm{N}=8 /$ genotype.

\section{Figures}



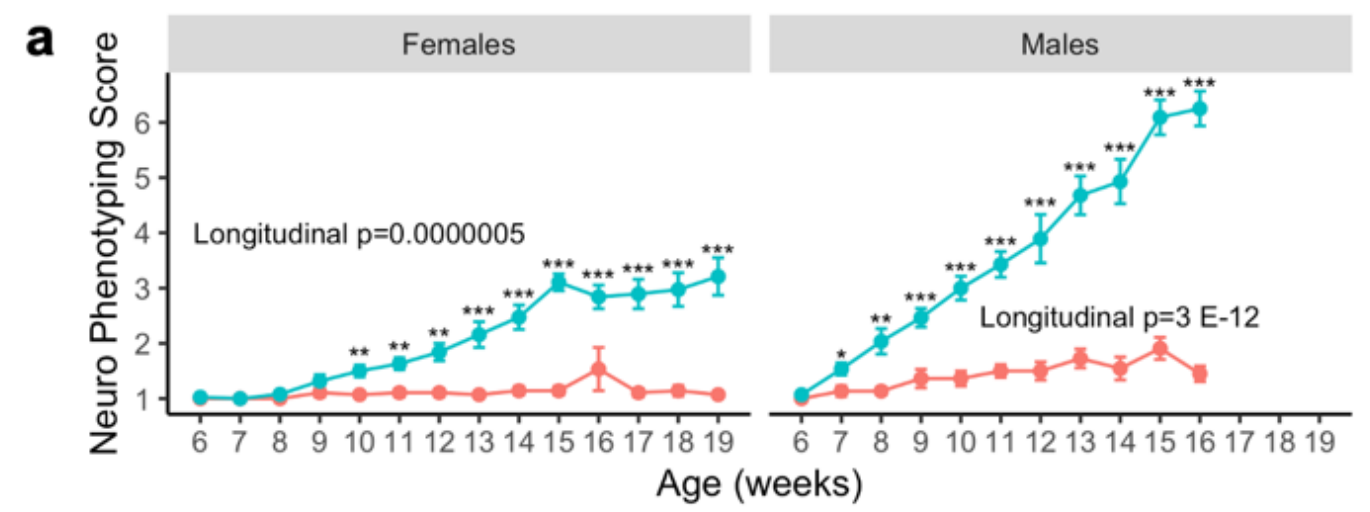

\section{Genotype \\ $\rightarrow$ mutant \\ $\rightarrow$ wild-type}

b

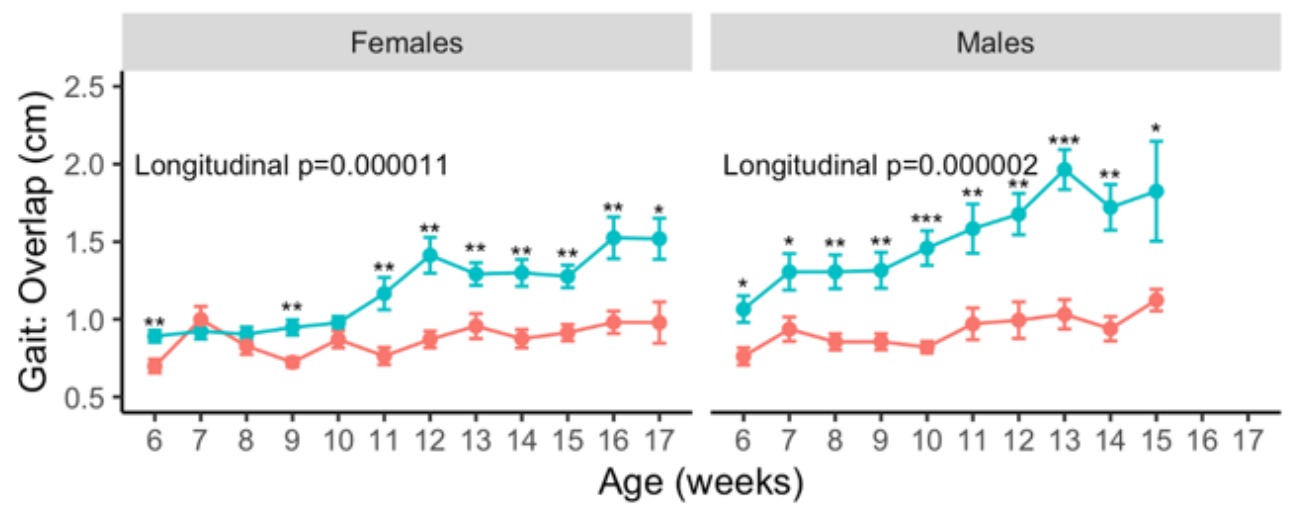

\section{Genotype}

$\rightarrow$ mutant

$\rightarrow$ wild-type

\section{C}

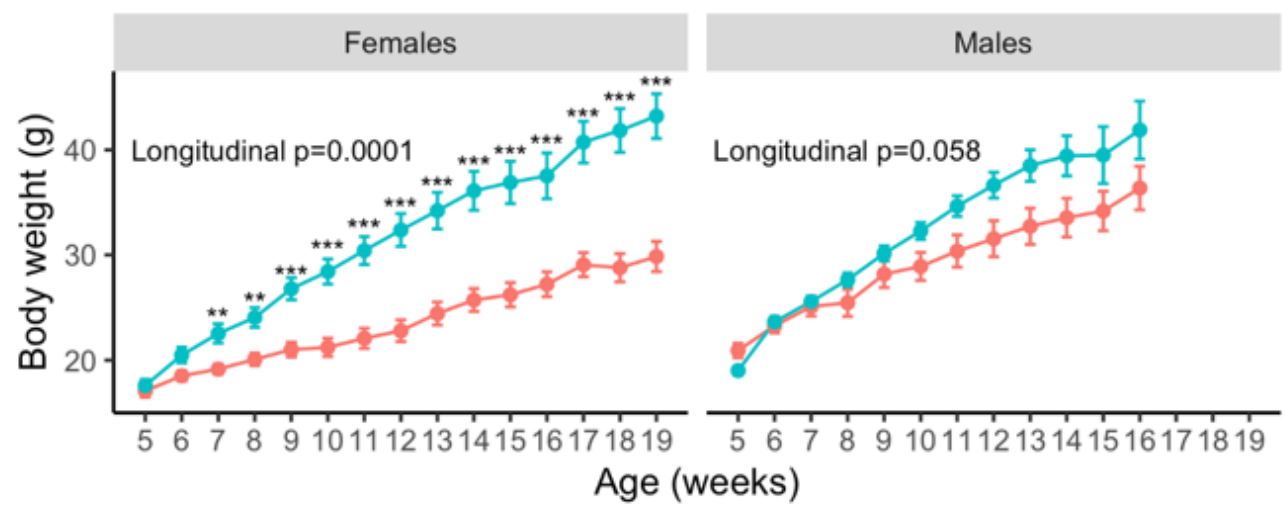

\section{Genotype}

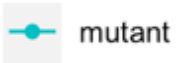

$\rightarrow$ wild-type

\section{Figure 1}

Longitudinal phenotypes in Mecp2-e1 mutant and wild-type mice. a) Comparison of neurophenotyping score (range 1-8, 1 being least severe and 8 being most severe) in Mecp2-e1 mutant vs. wild-type (wt) mice longitudinally and cross-sectionally across disease course (between 6 and 19 weeks of age for females and 6 and 16 weeks of age for males), stratified by sex and controlling for within-litter effects. b) Comparison of the most sensitive measure of gait (overlap distance in centimeters between hind and front legs, with a greater distance indicating a more impaired gait) in mutant vs. wild-type mice longitudinally and crosssectionally between 6 and 17 weeks of age in females and 6 and 15 weeks of age for males, stratified by sex and controlling for within-litter effects. c) Comparison of body weight of mutant vs. wild-type mice longitudinally and cross-sectionally between 5 and 19 weeks of age for females and 5 and 19 weeks of age for males, stratified by sex and controlling for within-litter effects. Cross-sectional analyses were controlled for multiple comparisons at each time point. N=11-19/genotype/sex. Each dot represents the mean of each group at each time point and error bars are standard error of the mean (SEM). *FDR $<0.05$, $\star \star F D R<0.01, \star \star \star F D R<0.001$ in mutant vs. control, cross-sectionally. Longitudinal p-values represent the overall association between mutant and wild-type mice across disease course using linear mixed effects models. 
a

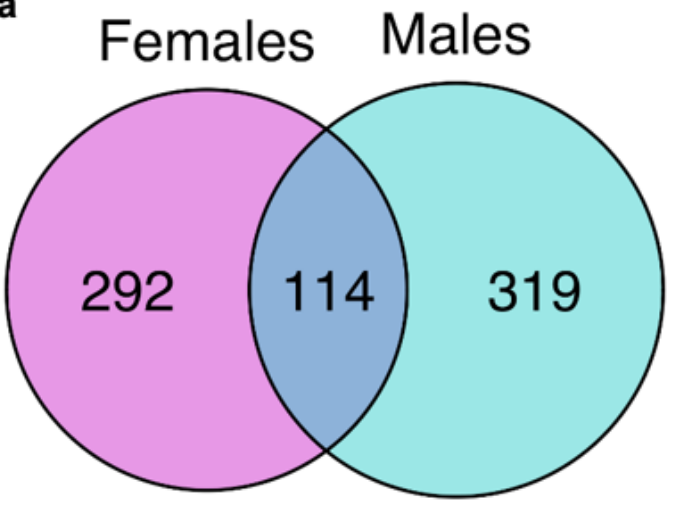

b Differentially Abundant Taxa in Mecp2-e1 Mutants vs. Wild-Type

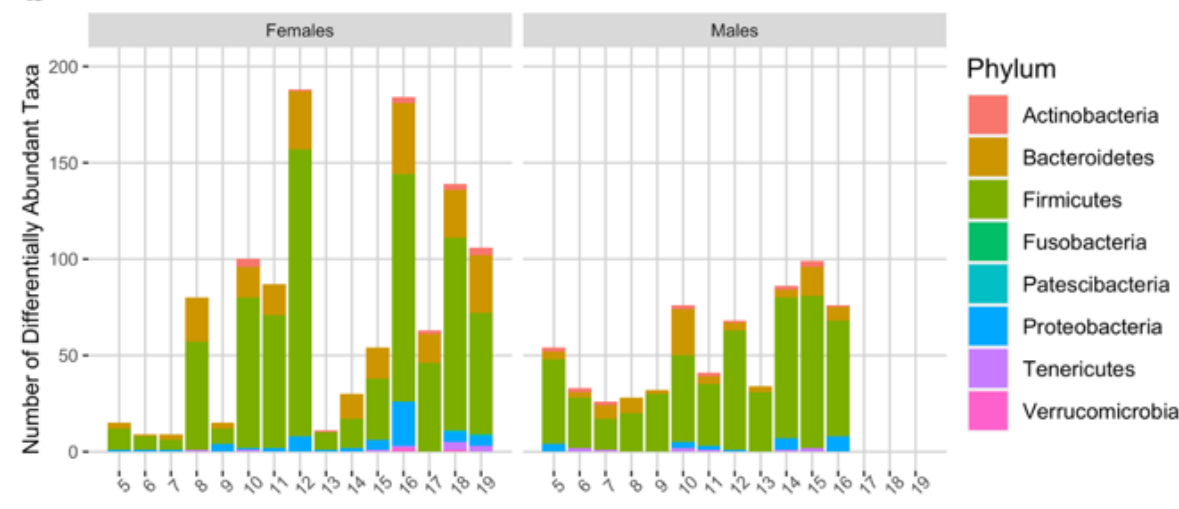

Age (weeks)

C Top 25 Genotype x Age ASVs

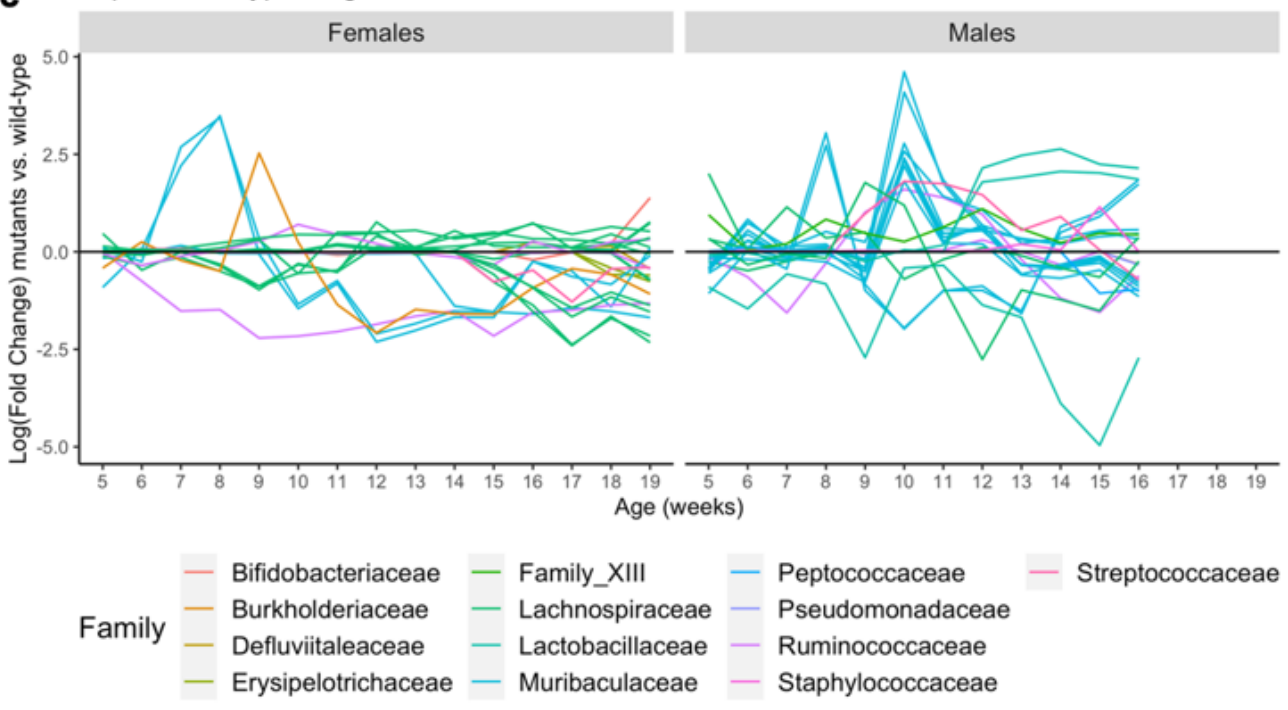

Figure 2

Longitudinal differences in the fecal microbiome in mutant vs. wild-type Mecp2-e1 mice. a) Venn diagram comparing ASVs that were significantly associated (FDR $<0.05$ ) with genotype longitudinally across disease course in females vs. males, while controlling for within-litter effects. b) Number of differentially abundant taxa (ASVs) by genotype in females and males crosssectionally at each time point between 5 and 19 weeks of age in females and 5 and 16 weeks of age in males, colored by phylum (FDR 0.05$)$. c) the top 25 ASVs with the most statistically significant genotype by age interaction plotted by log(fold change) in mutants vs. wild-type (wt) mice at each time point between 5 and 19 weeks of age in females and 5 and 16 weeks of age in males. Each line represents a single ASV and lines are colored by Family. N=11-19/genotype/sex. 

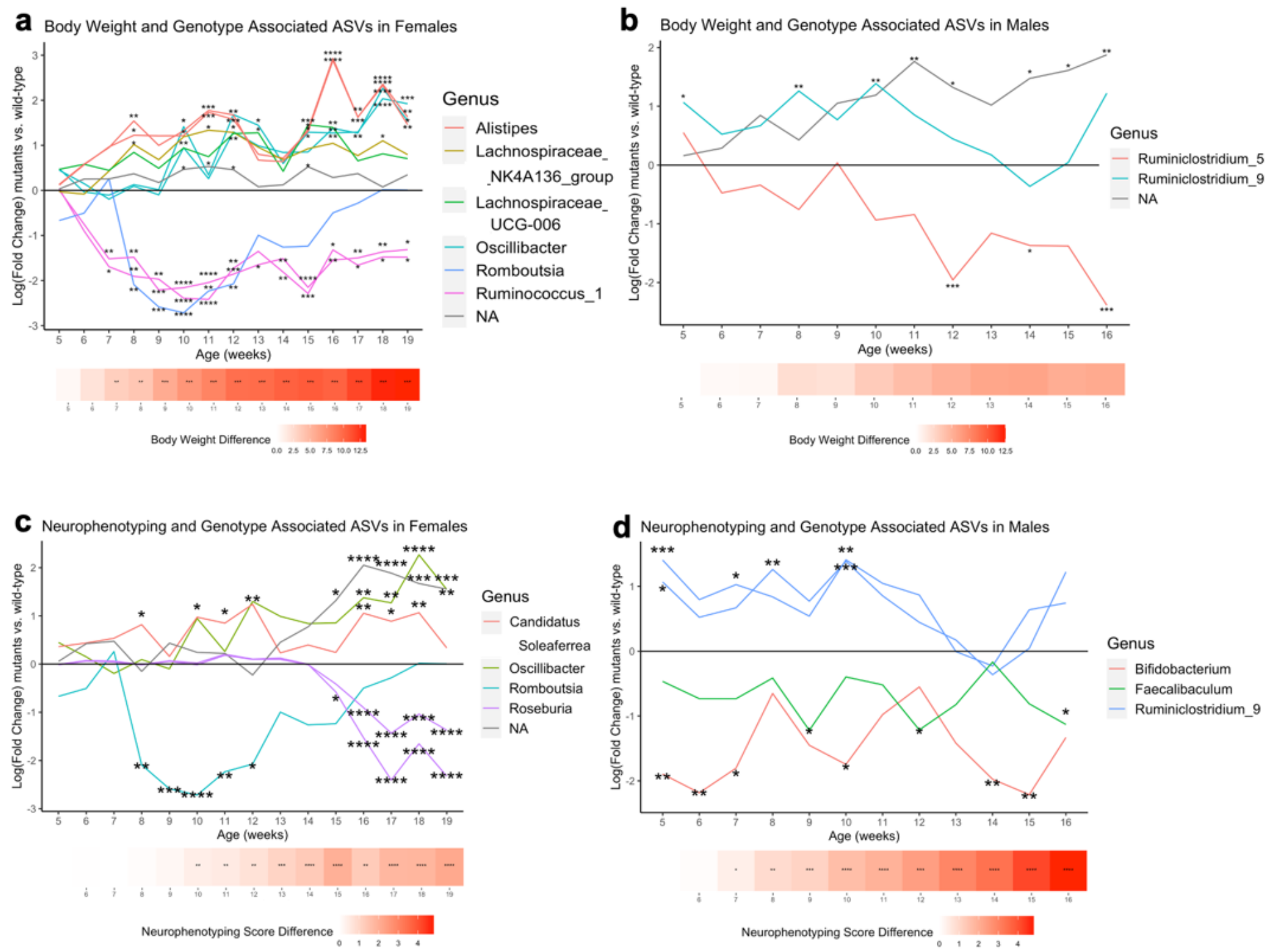

Figure 3

Differences in genotype- and phenotype-associated ASVs in mutant vs. wild-type Mecp2-e1 mice across disease course. Log(fold change) in normalized read counts of mutant vs. wild-type (wt) mice at each time point in ASVs that were significantly associated with phenotype and genotype at a minimum of one-quarter of the time points in both females and males. Each line represents an individual ASV and lines are colored by Genus. Heatmaps at the bottom of each graph are colored red with increasing intensity based on the difference in phenotype measure by genotype at each time point as reported in Figure 1. a) ASVs associated with both body weight and genotype in females, b) ASVs associated with both body weight and genotype in males, c) ASVs associated with both neurophenotyping score and genotype in females, d) ASVs associated with both neurophenotyping score

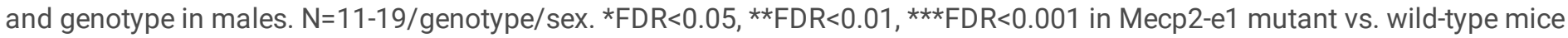
cross-sectionally at each time point. 


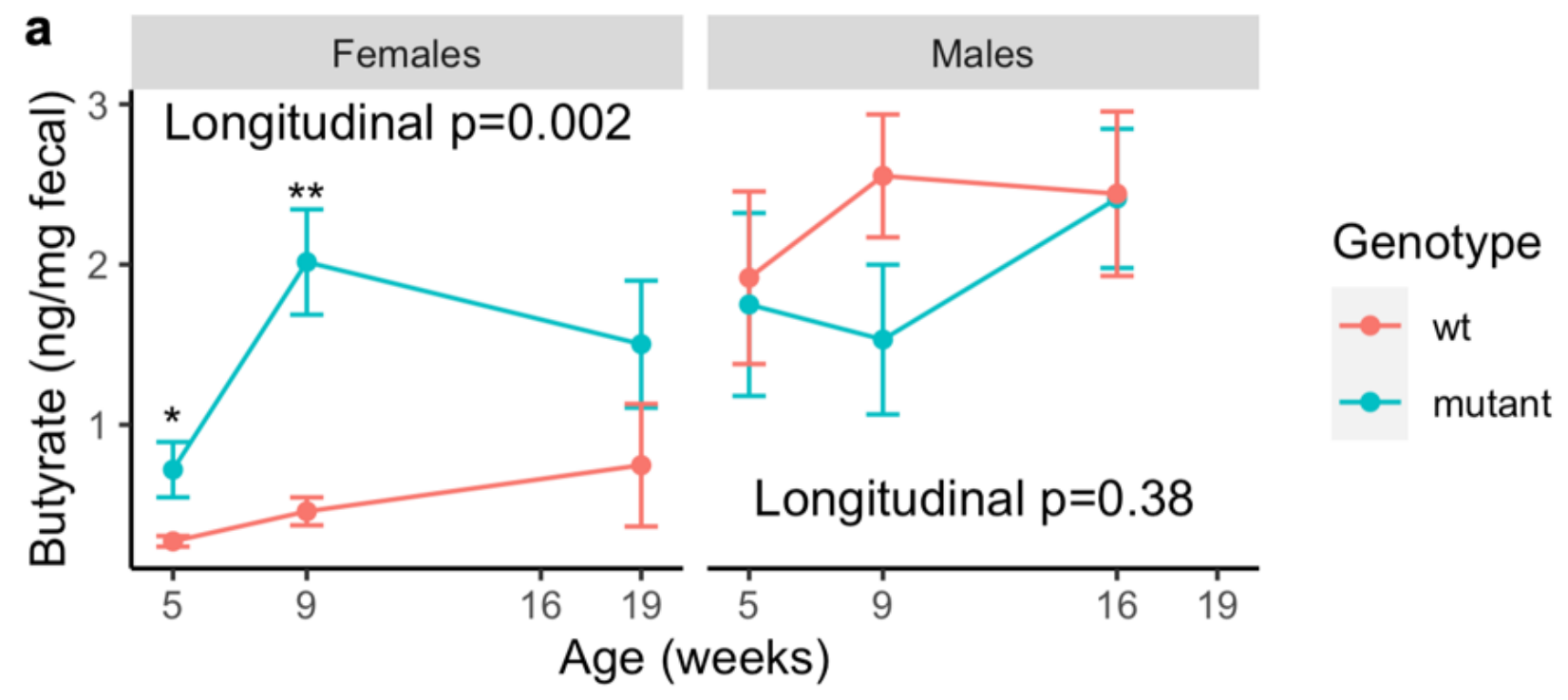

b

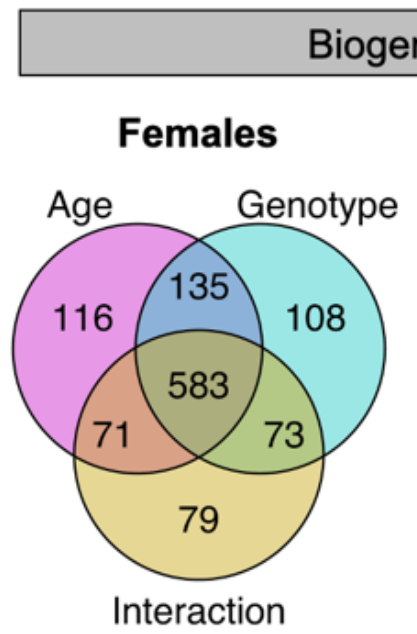

Biogenic Amines

Males

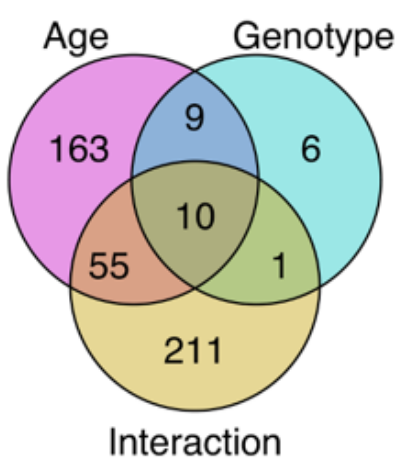

c

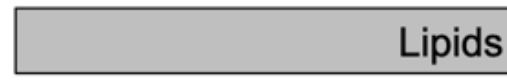

Females

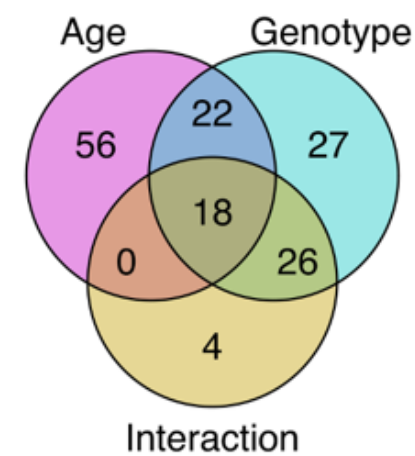

Males

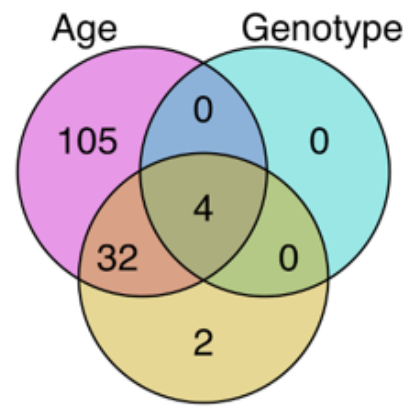

Interaction

Figure 4

Differentially abundant fecal metabolites by Mecp2-e1 genotype and age in female and male mice. a) Difference in butyrate concentrations in fecal samples in Mecp2-e1 mutant vs. wild-type (wt) mice longitudinally and cross-sectionally across disease course (at 5, 9, and 19 weeks of age in females and 5, 9, and 16 weeks of age in males), stratified by sex and controlling for within-litter effects. Each dot represents the mean of each group at each time point and error bars are standard error of the mean (SEM). $\mathrm{N}=8$ /genotype/sex. ${ }^{*} \mathrm{FDR}<0.05,{ }^{\star *} \mathrm{FDR}<0.01$ in Mecp2-e1 mutant vs. wild-type cross-sectionally. Longitudinal p-values represent the overall association between mutant and wild-type mice across disease course using linear mixed effects models. b) Venn diagrams comparing fecal biogenic amines and c) comparing fecal lipids that were longitudinally (at 5, 9, and 19 weeks for females and 5, 9, and 16 weeks for males) associated with age, genotype, and a genotype by age interaction (FDR $<0.05)$ in both females and males. $\mathrm{N}=8 /$ genotype/sex. 

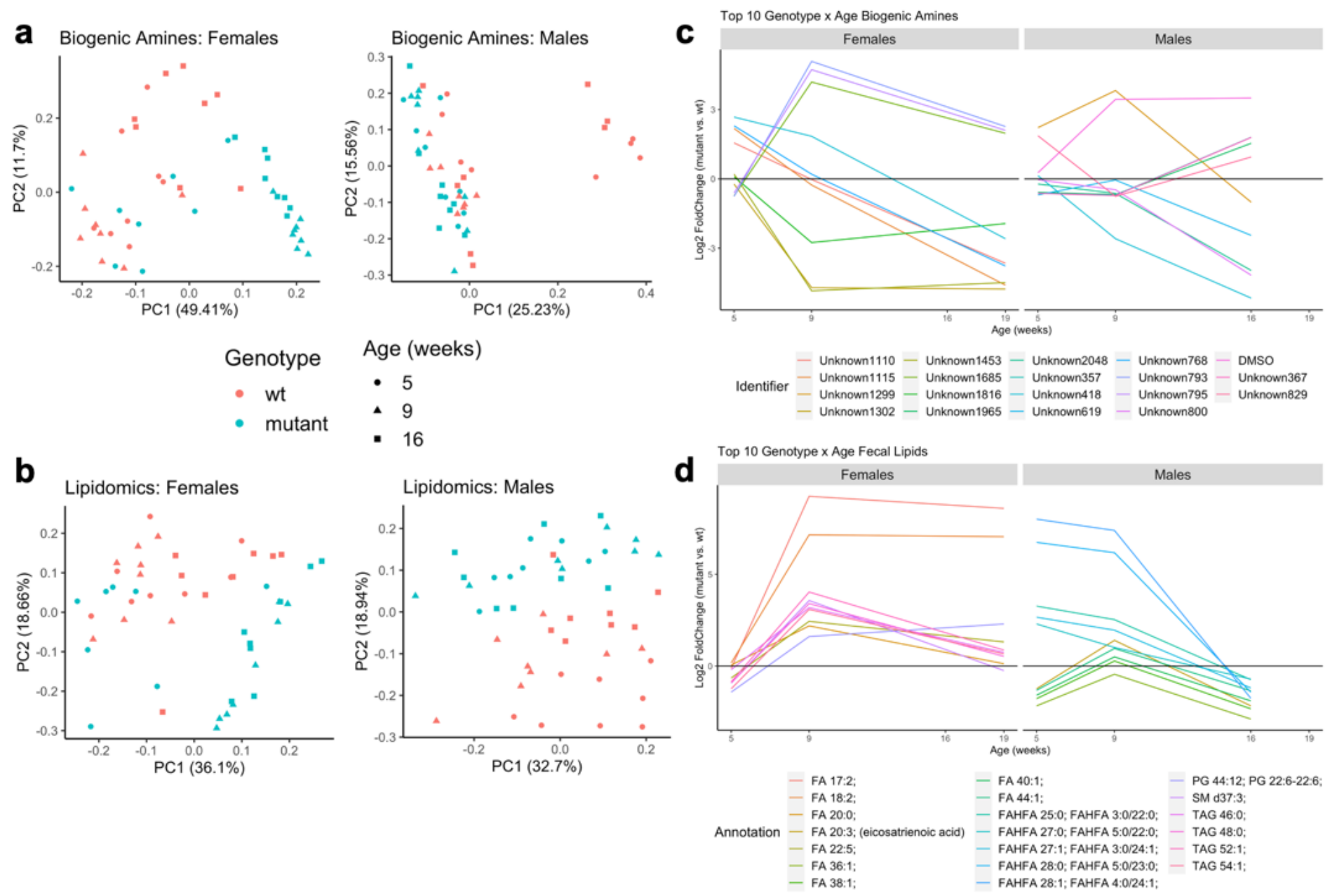

\section{Figure 5}

Longitudinal fecal metabolome profiles in mutant vs. wild-type Mecp2-e1 mice. Principal component analysis (PCA) plots of the top two principal components (PC1 and PC2) for fecal biogenic amines (a) and fecal lipids (b) with each point representing a single fecal sample and genotype represented by color and time point represented by shape. The log2(fold change) between mutant and wild-type (wt) for the top 10 biogenic amines (c) and lipids (d) with the most significant genotype by age interactions in females and males, plotted longitudinally across disease course (at 5, 9, and 19 weeks for females and 5, 9, and 16 weeks for males). Each line represents a single biogenic amine or lipid and is colored as such. FA = fatty acid, FAHFA = fatty acid esters of hydroxy fatty acid, TAG = triglyceride, $\mathrm{PG}=$ phosphatidylglycerol, $\mathrm{SM}=$ sphingomyelin. $\mathrm{N}=8 /$ genotype/sex. 

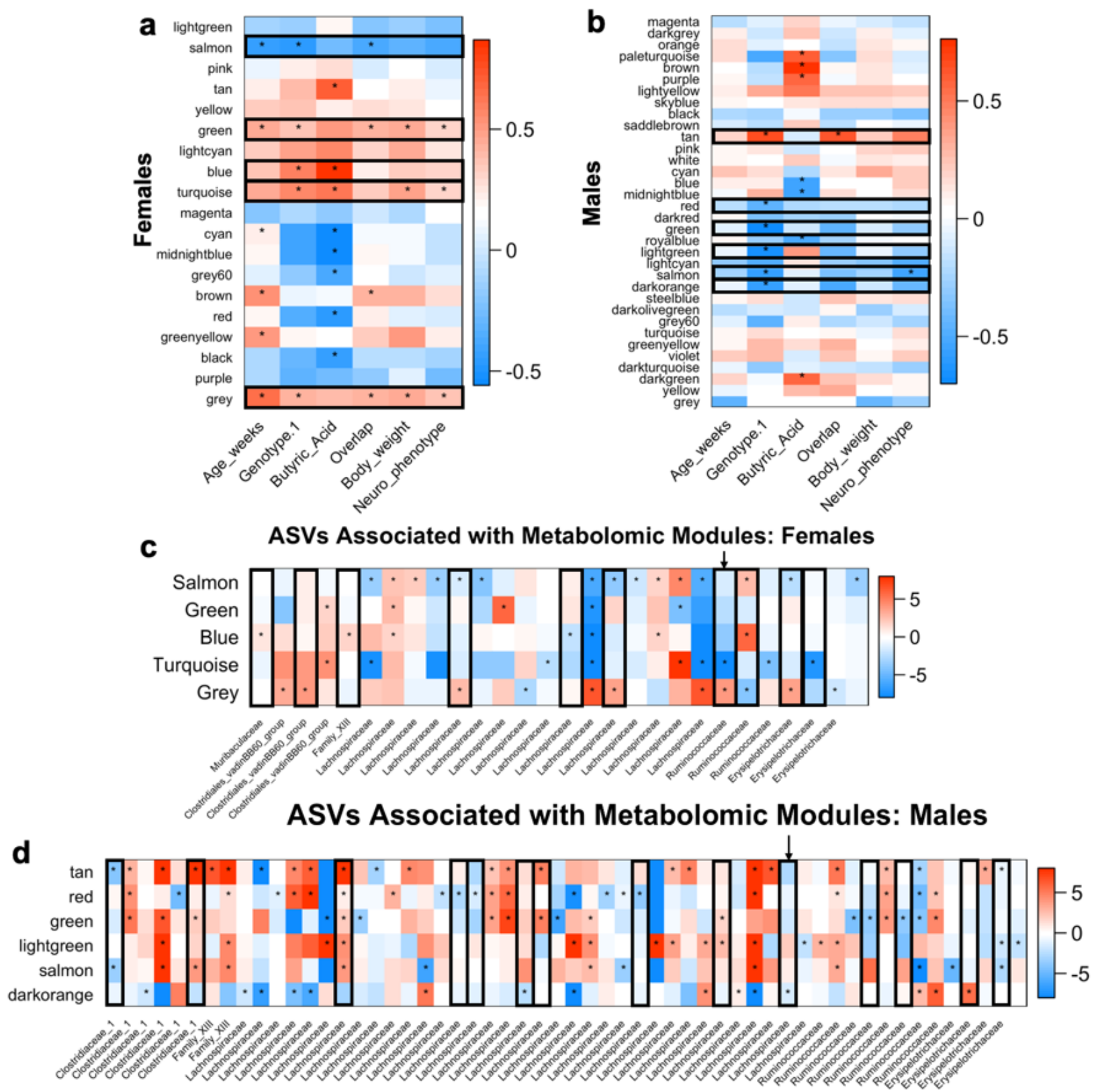

Figure 6

Relationships between the fecal metabolome and genotype, phenotype, and the fecal microbiome. Heatmaps depicting relationships between fecal metabolomic modules as constructed through weighted gene co-network analysis (WGCNA) and age, genotype, and phenotypes in females (a) and males (b). Blocks are colored by correlation coefficients with red representing positive correlations and blue representing negative correlations. Statistical analyses evaluating relationships between modules and traits were carried out via linear mixed effects models to control for within-litter effects and repeated measures. Relationships between genotype-associated metabolomic modules and fecal microbiota in females (c) and males (d) are depicted via heatmaps with blocks colored by log(fold change). Statistical analyses were carried out using limma controlling for within-litter effects and repeated measures. The only ASVs shown were those that were statistically significantly associated with at least one genotype-associated metabolomics module. $\mathrm{N}=8$ /genotype/sex. ${ }^{*} \mathrm{FDR}<0.05$. 


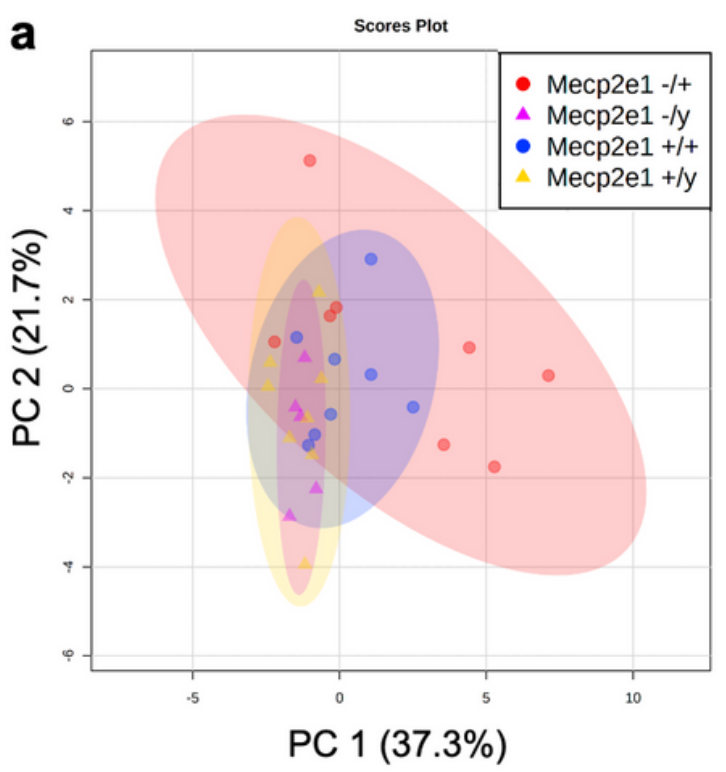

b Correlation Between Brain Lipids and Fecal Lipids at 9 weeks

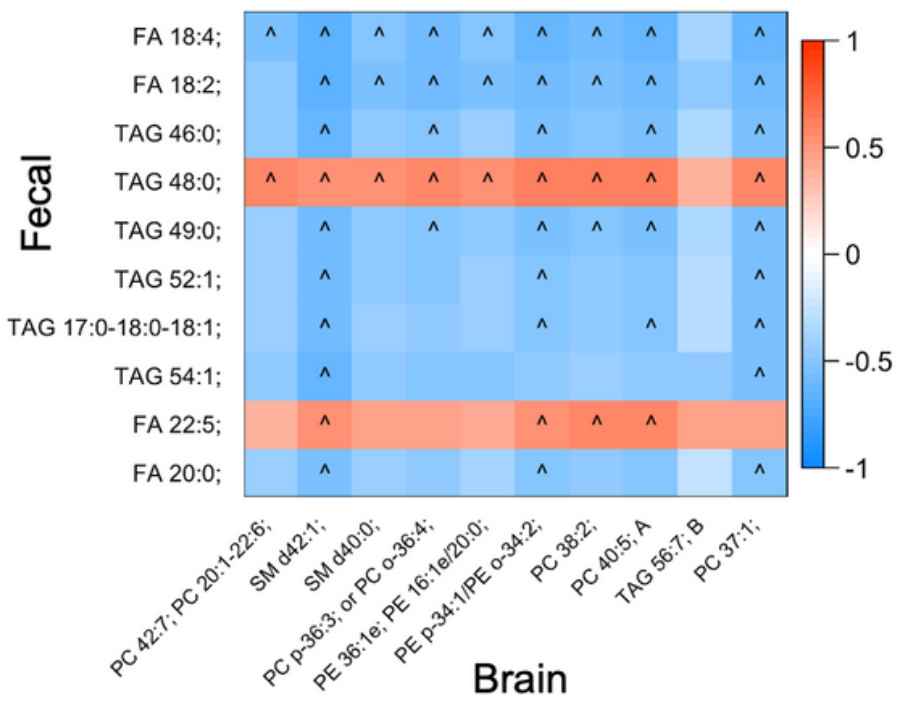

\section{Figure 7}

Brain lipidome profiles in Mecp2-e1 mutant and wild-type mice. a) Principal component analysis (PCA) plot of female and male mutant and wild-type cortical lipids measured at the end of disease course (19 weeks for females and 16 weeks for males). The two principal components (PCs) that explain the most variation in samples are plotted with PC 1 on the x-axis and PC 2 on the $y$ axis. Each dot represents one sample, with colors representing genotype and shapes representing sex. b) Heatmap depicting the relationship between the 10 fecal lipids and the 10 brain lipids with the most significant association with genotype in females. Associations were strongest for fecal lipids at 9 weeks of age, and thus those are depicted here. Blocks are colored based on correlation coefficient, with red representing positive correlations and blue representing negative correlations. $\mathrm{N}=6$ 8/genotype/sex. ^ FDR<0.10. 


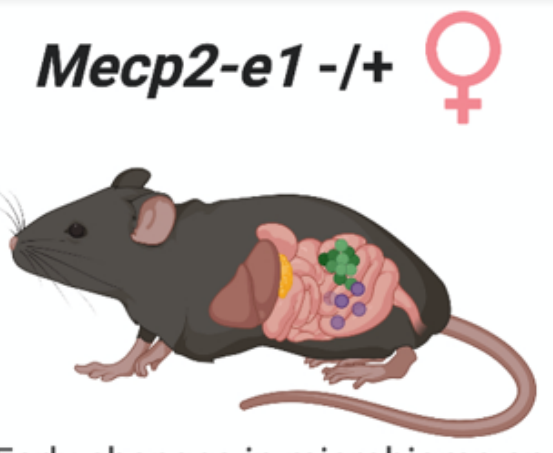

Early changes in microbiome and metabolome
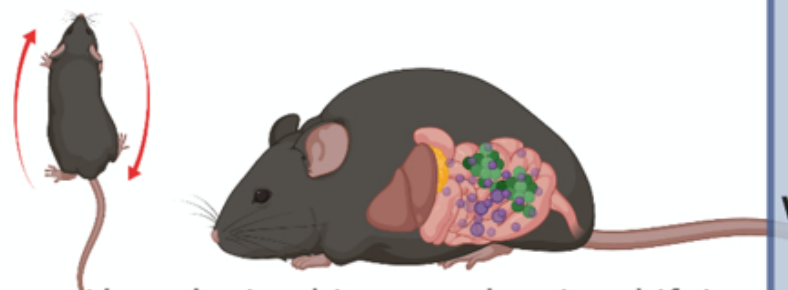

Altered microbiome and major shift in metabolome, altered gait and neurophenotyping score, increased body weight

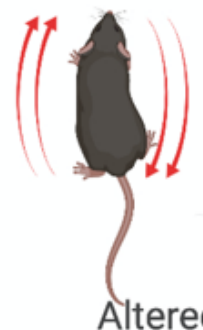

Altered microbiome and metabolome, worsened gait and neurophenotyping score, progressively increased body weight, altered brain lipidome

\section{Mecp2-e1-/y O"}

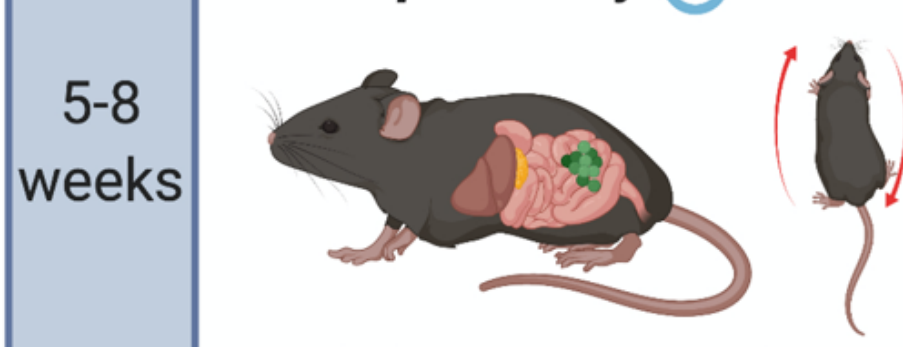

Early changes in gut microbiome, altered gait and neurophenotyping score

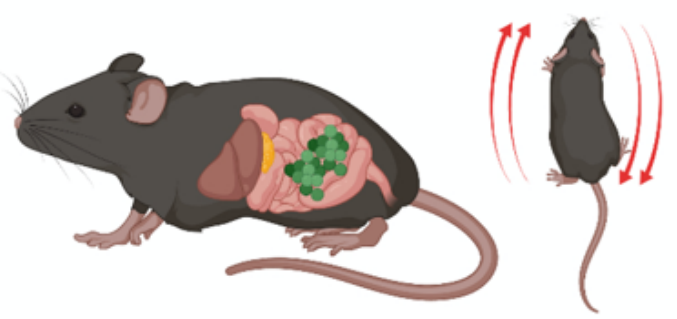

Altered microbiome, progressively worsened gait and neurophenotyping score

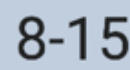

weeks

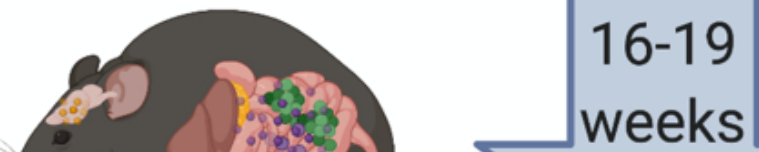

weeks

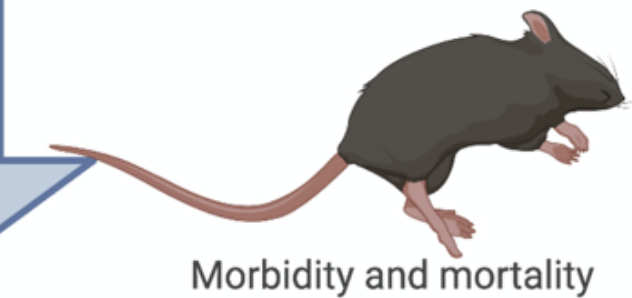

\section{Figure 8}

Female and male Mecp2-e1 mutants exhibit divergent phenotypic and molecular RTT disease progression. In early adulthood at 5-7 weeks of age, Mecp2-e1 mutant males begin to show declines in motor and neurological function and changes in their gut microbiome. On the other hand, female Mecp2-e1 mutants at 5-8 weeks of age do not yet display motor and neurological symptoms, but begin to show increased body weight, altered gut microbiota and altered gut metabolites, such as short chain fatty acids (SCFAs). Females begin to display progressively increased body weight and drastic shifts in the gut metabolome at 9 weeks of age, prior to persistent declines in neurological and motor function which occurred at 10-11 weeks of age. Peak differences in the gut microbiome occurred at 12 weeks of age in females. Males, on the other hand, display progressive worsening of neuromotor symptoms and progressive changes in the gut microbiome in the absence of drastic shifts in the gut metabolome between 9 and 15 weeks of age. In late-stage RTT disease between 16 and 19 months of age, males exhibit severe morbidity and early mortality, whereas females continue to display progressively increased body weight and neuromotor function, continued evidence of a disrupted gut microbiome and metabolome, and altered brain lipid profiles.

\section{Supplementary Files}


This is a list of supplementary files associated with this preprint. Click to download.

- SupplementalFigures.pdf

- SupplementalTables115.xlsx 